\title{
The Ty1 integrase nuclear localization signal is necessary and sufficient for retrotransposon targeting to tRNA genes
}

\section{Authors}

Amna Asif-Laidin ${ }^{1}$, Christine Conesa ${ }^{2,3}$, Amandine Bonnet ${ }^{1,3}$, Camille Grison ${ }^{1}$, Indranil Adhya $^{2}$, Rachid Menouni ${ }^{1}$, Hélène Fayol ${ }^{1}$, Noé Palmic ${ }^{1}$, Joël Acker ${ }^{2, *}$ and Pascale Lesage $^{1,4, *}$

\section{Affiliations}

${ }^{1}$ INSERM U944, CNRS UMR 7212, Genomes \& Cell Biology of Disease Unit, Institut de Recherche Saint-Louis, Université de Paris, Hôpital Saint-Louis, Paris, France.

${ }^{2}$ Institute for Integrative Biology of the Cell (I2BC), CEA, CNRS, Univ. Paris-Sud, Université Paris-Saclay, 91198 Gif-sur-Yvette cedex, France.

\author{
Author list footnotes \\ ${ }^{3}$ Equal contribution \\ ${ }^{4}$ Lead contact \\ *Correspondence: pascale.lesage@inserm.fr, joel.acker@cea.fr
}




\section{SUMMARY}

Integration of transposable elements into the genome is mutagenic. Mechanisms that target integration into relatively safe locations and minimize deleterious consequences for cell fitness have emerged during evolution. In budding yeast, the integration of the Ty1 LTR retrotransposon upstream of RNA polymerase III (Pol III)-transcribed genes requires the interaction between the AC40 subunit of Pol III and Ty1 integrase (IN1). Here we show that the IN1-AC40 interaction involves a short linker sequence in the bipartite nuclear localization signal (bNLS) of IN1. Mutations in this sequence do not impact the frequency of Ty1 retromobility, instead they decrease the recruitment of IN1 to Pol III-transcribed genes and the subsequent integration of Ty1 at these loci. The replacement of Ty5 retrotransposon targeting sequence by the IN1 bNLS induces Ty5 integration into Pol III-transcribed genes. Therefore, the IN1 bNLS is both necessary and sufficient to confer integration site specificity on Ty1 and Ty5 retrotransposons.

\section{KEY WORDS}

Transposable element, Ty1 retrotransposon, integration targeting, RNA Polymerase III, nuclear localization signal 


\section{INTRODUCTION}

Transposable elements (TEs) are mobile repetitive DNA sequences found in the genomes of most organisms (Huang et al., 2013). TEs are mutagenic and represent a threat to genome integrity, inactivating or altering host gene expression or inducing large chromosomal rearrangements (Bourque, 2009; Levin and Moran, 2011). In humans, more than hundred heritable diseases have been assigned to de novo TE insertions (Hancks and Kazazian 2016). TEs also play a role in genome evolution by modifying host functions, phenotypes, and gene regulation, and can contribute to the long-term adaptation of organisms to different environments (Chuong et al., 2016).

Where TEs integrate in the genome will determine their impact on their host. TE distribution, which is rarely random (Sultana et al., 2017), arises from the balance between two processes. First, selection leads to the elimination of strongly deleterious insertions, and the maintenance of beneficial ones (Chuong et al., 2016; Cosby et al., 2019). Second, TEs have repeatedly evolved mechanisms that direct their integration into "safe" locations, where insertions will have minimal adverse effect on the organism's fitness (Boeke and Devine, 1998; Cheung et al., 2018; Spaller et al., 2016). These regions often consist of non-essential repeated sequences, such as telomeric regions, ribosomal DNA arrays, and transfer RNA genes $(t D N A \mathrm{~s})$, or non-essential regions upstream of open reading frames (Baller et al., 2012; Fujiwara et al., 2005; Guo and Levin, 2010; Kling et al., 2018; Mularoni et al., 2012; Naito et al., 2009; Pardue and DeBaryshe, 2011; Penton and Crease, 2004; Ye et al., 2005; Zou et al., 1996). Preferential targets have been described for the integration of different classes of TEs, including retroelements (Sultana et al., 2017).

Long terminal repeat (LTR) retrotransposons are retroelements related to retroviruses. They replicate by reverse transcription of their mRNA into a double-stranded DNA copy (cDNA), which is imported into the nucleus and integrated into the genome by the LTRretrotransposon integrase (IN). The interaction between IN and cellular tethering factors plays a major role in integration site selection by targeting pre-integration complexes (PICs) to specific chromosome locations. Tethering factors were first identified for Ty 3 and Ty 5 in $S$. cerevisiae. These LTR retrotransposons integrate at the transcription start site of Pol IIItranscribed genes and in subtelomeric regions, respectively (Kirchner et al., 1995; Xie et al., 2001). The Tf1 LTR retrotransposon of Schyzosaccharomyces pombe and the MLV retrovirus both target the promoter region of Pol II-transcribed genes (Gupta et al., 2013; Hickey et al., 
2015; Jacobs et al., 2015; De Rijck et al., 2013; Sharma et al., 2013), and the HIV-1 retrovirus targets the gene body of Pol II-transcribed genes (Cherepanov et al., 2003; Llano et al., 2006). In all cases, tethering factors bind chromatin or have functions related to DNA transcription or replication (Sultana et al., 2017).

Ty1, the most active and abundant LTR-retrotransposon in S. cerevisiae, integrates preferentially within a $1 \mathrm{~kb}$ window upstream of Pol III-transcribed genes. It targets nucleosomal DNA near the H2A/H2B interface (Baller et al., 2012; Mularoni et al., 2012). This integration pattern allows Tyl to replicate while minimizing disruption to the host genome, as most Pol III-transcribed genes are multicopy $t D N A \mathrm{~s}$ and thus individually nonessential. Furthermore, Ty1 insertion has a limiting impact on $t D N A$ expression (Bolton and Boeke, 2003). Targeted integration proximal to $t D N A \mathrm{~s}$ is a strategy that has been adopted several times by TEs to minimize damage to compact genomes (Cheung et al., 2018; Kling et al., 2018). The integration of Ty1 in these regions requires a functional Pol III promoter in the target gene (Devine and Boeke, 1996) and is influenced by the chromatin-remodeling factor Isw2 and the Bdp1 subunit of TFIIIB (Bachman et al., 2005).

Recently, we have shown that an interaction between Ty1 IN (IN1) and the AC40 subunit of Pol III is a major driver for Ty1 integration upstream of Pol III-transcribed genes (BridierNahmias et al., 2015). The study used the $S$. pombe AC40 ortholog (AC40sp) as a loss-ofinteraction mutant. The replacement of AC40 by AC40sp severely compromised Ty1 integration upstream of Pol III-transcribed genes, leading to a redistribution of Ty1 insertions in the genome. IN1 binding to other Pol III subunits was also described in vitro (Cheung et al., 2016). However, it is not clear whether these interactions participate to Tyl integration site selection.

IN1 has a three domains organization common to all retroelement integrases; the $\mathrm{Zn}^{2+}$ coordinating N-terminal domain (NTD), the catalytic core domain (CCD) and the less conserved C-terminal domain (CTD) (Figure 1A, top) (Wilhelm et al., 2005). IN1 C-terminal residues 578-635 are necessary and sufficient to mediate the interaction with AC40 in vivo (Bridier-Nahmias et al., 2015). This region also contains a bipartite nuclear localization signal (bNLS, residues 596-630; Figure 1A, top) consisting of two Lys-Lys-Arg motifs separated by a 29 amino-acid linker (Kenna et al., 1998; Lange et al., 2011; Moore et al., 1998). This raises the question of whether IN1 nuclear import and interaction with AC40 could act in concert during Ty1 replication. 
In this study, we identify a short sequence in the bNLS linker of IN1 that directs the interaction with AC40. Single amino acid substitutions in this sequence do not affect the frequency of Ty1 retrotransposition but impair the recruitment of IN1 to Pol III-transcribed genes. Consequently, these IN1 mutations induce the same changes in the Ty1 integration profile as observed in the AC40sp loss-of-interaction mutant. When the IN1 bNLS is used to replace the Ty5 IN sequence responsible for Ty5 integration into heterochromatin, Ty5 integration is re-directed to Pol III-transcribed genes. This work therefore confirms the fundamental role of the IN1-AC40 interaction in Ty1 integration site selection and reveals that the IN bNLS is necessary and sufficient to confer Tyl integration preference to another retrotransposon. 


\section{RESULTS}

\section{bNLS linker sequence mutations abolish the interaction with AC40.}

S. cerevisiae LTR-retrotransposons Ty1, Ty2, and Ty4 have the same integration preferences for regions upstream of Pol III-transcribed genes (Carr et al., 2012; Kim et al., 1998) and the C-termini of their integrases (IN1, IN2, and IN4, respectively) interact with the Pol III subunit AC40 (Bridier-Nahmias et al., 2015). To identify conserved amino acids potentially involved in the AC40 interaction, we aligned the C-terminal sequences of IN1, IN2, and IN4 (Figure $1 \mathrm{~A}$, bottom) and observed that IN1 and IN2 are highly similar in this region, whereas IN4 is more divergent. Amino acids at positions 600-601, 609-610, and 617-623 in IN1 were either identical or highly similar in all three INs. We replaced each of these amino acids by alanine, individually or in pairs, in a Gal4 activating domain GAD-IN $1_{578-635}$ fusion protein and studied the interaction of the mutant fusion proteins with $\underline{\text { Gal4 }}$ binding domain GBD-AC40 using a two-hybrid assay. The interaction between $\mathrm{IN}_{\text {578-635 }}$ and AC40 was maintained in the presence of mutations $\mathrm{LE}_{600-601} \mathrm{AA}, \mathrm{VS}_{609-610} \mathrm{AA}, \mathrm{N}_{618} \mathrm{~A}$ or $\mathrm{E}_{623} \mathrm{~A}$, and suppressed by single alanine substitution of $\mathrm{K}_{617}, \mathrm{M}_{619}, \mathrm{R}_{620}, \mathrm{~S}_{621}$ or $\mathrm{L}_{622}$ (Figure 1B). GBD-AC40 interacted with GAD-IN1 $1_{578-635}$ but not with GAD-IN1 1-578, as shown previously (Bridier-Nahmias et al., 2015). Since amino acids $\mathrm{K}_{617}-\mathrm{L}_{622}$ are located in the IN1 bNLS linker sequence, we also tested the interaction between GBD-AC40 and GAD fused to the entire bNLS sequence (GAD-IN $\left.{ }_{596-630}\right)$. Interaction between the two fusion proteins was detected, suggesting that this region of 34 amino acids in IN1 is required for interaction with AC40 (Figure 1C).

To determine whether amino acids required for the two-hybrid interaction between AC40 and the IN1 C-terminus were also critical for the interaction between the two full-length proteins, we co-expressed AC40-strep and WT or mutant IN1-EPEA (Glu-Pro-Glu-Ala) tagged proteins in E. coli and performed immunoprecipitation of the purified proteins. We focused on $\mathrm{K}_{617}, \mathrm{~S}_{621}$ and $\mathrm{L}_{622}$, given their strict conserved in IN1, IN2 and IN4 (Figure 1A, bottom). In the presence of Strep-Tactin beads, AC40-strep co-immunoprecipitated with WT IN1EPEA but not with the IN1-EPEA $\mathrm{K}_{617} \mathrm{~A}, \mathrm{~S}_{621} \mathrm{~A}$, and $\mathrm{L}_{622} \mathrm{~A}$ mutants (Figure 1D). Thus, fulllength IN1 and AC40 proteins bind directly to each other, and their interaction depends on residues $\mathrm{K}_{617}, \mathrm{~S}_{621}$ and $\mathrm{L}_{622}$ located in the IN1 bNLS. 


\section{Non-AC40 binding IN1 mutants do not affect Ty1 integration frequency.}

Mutations in the IN1 bNLS that induce a substantial or complete loss of IN1 nuclear accumulation reduce the frequency of Ty1 retromobility, as seen when the two Lys-Lys-Arg (KKR) motifs are mutated, either individually or simultaneously, but also for mutations of specific acidic residues in the linker region (Kenna et al., 1998; Lange et al., 2011; Moore et al., 1998). To investigate whether the bNLS amino acids we identified as being necessary for the AC40 interaction were also required for Tyl nuclear import and retromobility, we introduced $\mathrm{K}_{617} \mathrm{~A}, \mathrm{~S}_{621} \mathrm{~A}$ or $\mathrm{L}_{622} \mathrm{~A}$ mutations into a $\mathrm{GFP}_{2}$-bNLS fusion protein previously used to assess IN1 bNLS function (McLane et al., 2008). In contrast to the IN1-bNLSmut construct ( ${ }_{596} \mathrm{KKR}_{598}-\mathrm{AAA}$ and ${ }_{628} \mathrm{KKR}_{630}$-AAA), the three IN1-bNLS single mutants were still able to target $\mathrm{GFP}_{2}$ into the nucleus (Figure 2A). Thus, amino acids required for the IN1-AC40 interaction are dispensable for NLS function.

The same mutations were introduced individually into a Tyl element containing the retromobility indicator gene his $3 A I$, allowing detection of Ty1-HIS3 insertion events as $\mathrm{His}^{+}$ prototroph cells and expressed from the GAL1 promoter in a 2-micron plasmid (Curcio and Garfinkel, 1991). To determine the frequency of Tylhis $3 A I$ integration in the genome, we expressed this plasmid in a spt3-101 null rad52 $\Delta$ mutant strain, deficient in endogenous Ty1 expression and homologous recombination. SPT3 is required for Ty1 transcription and its absence prevents the trans-complementation of the mutant IN1 by WT IN1 from endogenous Ty1 elements (Winston et al., 1984). RAD52 deletion precludes insertion of the Ty1-HIS3 cDNA by homologous recombination with preexisting genomic Tyl copies, a preferred pathway when IN1-dependent integration is defective (Sharon et al., 1994). The frequency of $\mathrm{His}^{+}$cells was similar between strains expressing WT or $\mathrm{K}_{617} \mathrm{~A}, \mathrm{M}_{619} \mathrm{~A}, \mathrm{R}_{620} \mathrm{~A}, \mathrm{~S}_{621} \mathrm{~A}$ or $\mathrm{L}_{622} \mathrm{~A}$ mutant Ty1his3AI (Figure 2B). In contrast, mutations that inactivate IN1 nuclear import (Moore et al., 1998) or catalytic activity (Wilhelm and Wilhelm, 2005) caused a substantial decrease in the frequency of $\mathrm{His}^{+}$cells compared to WT (Figure 2B, mutants $\mathrm{KKR}_{628-630} \mathrm{GGT}$ and $\mathrm{D}_{154} \mathrm{~A}$, respectively).

Thus, single amino acid mutations in the linker of the IN1 bNLS that prevent the interaction with AC40 do not impair Ty1 retrotransposition. These data confirm that the IN1-AC40 interaction is not required for Tyl overall integration frequency (Bridier-Nahmias et al., 2015). 


\section{AC40 recruits IN1 at Pol I and Pol III-transcribed genes.}

In addition to AC40, other Pol III subunits have been suggested to mediate the interaction between IN1 and Pol III (Cheung et al 2016). To determine whether IN1 remains associated with Pol III in the absence of the IN1-AC40 interaction, we immunoprecipitated Pol III (Oficjalska-Pham et al., 2006) in yeast cells expressing hemagglutinin (HA)-tagged C160, the largest Pol III subunit, and WT or mutant IN1 fused to streptavidin (IN1-strep). WT IN1 was associated to Pol III but not the $\mathrm{K}_{617} \mathrm{~A}, \mathrm{~S}_{621} \mathrm{~A}$ or $\mathrm{L}_{622} \mathrm{~A}$ IN1 mutants (Figure $3 \mathrm{~A}$ ). Therefore, the interaction with AC40 is necessary for IN1 binding to Pol III in vivo.

To determine if AC40 plays a major role in IN1 recruitment to Pol III-transcribed genes, we developed IN1 chromatin-immunoprecipitation (ChIP) experiments to assay the effect of the $\mathrm{K}_{617} \mathrm{~A}, \mathrm{~S}_{621} \mathrm{~A}$, and $\mathrm{L}_{622} \mathrm{~A}$ mutations on recruitment. WT IN1 and the various mutants were tagged at their N-terminus using a 3xHA epitope tag and expressed from a tetracycline-off promoter. Quantitative PCR revealed significant enrichment of ectopic WT HA-IN1 at all tested Pol III-transcript loci, compared to background level measured on the GAL1 gene promoter (Figure 3B). In contrast, HA-IN1 mutants that did not interact with AC40 were barely detected at these loci. Thus, recruitment of IN1 to Pol III-transcribed loci depends on its interaction with AC40.

To assess if the genome-wide occupancy of WT IN1 correlates with Ty1 integration site preferences, we performed ChIP sequencing (ChIP-seq) using the same HA-IN1 constructs, with an untagged IN1-expressing strain as control. Analysis of reads mapping to unique sites revealed a strong association of WT HA-IN1 with most nuclear $t D N A s$ and the Pol IIItranscribed genes $S N R 6, S N R 52, S C R 1, R P R 1$, and RDN5. Very weak or no HA-IN1 binding was observed for RNA170 and ZOD1, previously shown to have low level of Pol III occupancy (Moqtaderi and Struhl, 2004) (Figure 3C and Table S1). Three $t D N A \mathrm{~s}, t K(C U U) C$, $t M(C A U) C$, and $t D(G U C) N$, were not recovered: These genes are either absent or transcriptionally inactive in the laboratory strain we used (Kumar and Bhargava, 2013; Patterson et al., 2019). HA-IN1 was absent from most Pol II-transcribed genes (Table S1) or present at a much lower level than at Pol III-transcribed genes (Figure 3C). Low HA-IN1 occupancy at Pol II transcribed-genes may be an artifact of ChIP-seq due to the level of expression of these genes (Teytelman et al., 2013). We did not detect significant HA-IN1 binding at other chromosomal loci. The genome-wide distribution of ectopic WT IN1 revealed a strong bias for Pol III-transcribed genes, confirming that the interaction of IN1 
with the Pol III is the main driver for targeted integration of Ty1. Under physiological conditions, IN1 is associated with Ty1 cDNA as part of the PIC. In our experimental conditions, IN1 was expressed ectopically at $30^{\circ} \mathrm{C}$, a temperature that restricts Ty1 replication. Therefore, our results indicate that the cDNA is not necessary for IN1 recruitment at Pol III. A recent study reached a similar conclusion for the recruitment of Ty3 integrase at $t D N A$ genes (Patterson et al., 2019).

ChIP-seq analysis of the HA-IN1 mutants that compromise the IN1-AC40 interaction $\left(\mathrm{K}_{617} \mathrm{~A}\right.$, $\mathrm{S}_{621} \mathrm{~A}$, and $\mathrm{L}_{622} \mathrm{~A}$ ) revealed their occupancy was substantially reduced at all Pol III-transcribed genes, as indicated by the lower number of reads corresponding to Pol III-transcribed genes with the three mutants, compare to WT HA-IN1 (Figure 3D), and by a metagene analysis comparing WT and mutant HA-IN1 binding on all the 275 nuclear $t D N A s$ (Figure 3E). No similar effect of these mutants was observed at Pol II-transcribed genes (Figure 3F). Quantification by pair-wise Spearman correlation between WT and either $\mathrm{K}_{617} \mathrm{~A}, \mathrm{~S}_{621} \mathrm{~A}$, or $\mathrm{L}_{622} \mathrm{~A}$ IN1 confirmed the apparent stronger decrease in Pol III occupancy of $\mathrm{K}_{617} \mathrm{~A}$ HA-IN1 compared to the other mutants (Figures 3E and S2A-B). The metagene analysis indicated a sharp peak around the transcription start site (TSS), which does not coincide with Ty1 integration sites, normally located upstream of Pol III-transcribed genes (Baller et al., 2012; Bridier-Nahmias et al., 2015; Mularoni et al., 2012). This suggests that in addition to the interaction with Pol III, other features may determine Ty1 integration site preference. HA-IN1 occupancy was not completely suppressed in the three mutants, suggesting that the $\mathrm{K}_{617}, \mathrm{~S}_{621}$ or $\mathrm{L}_{622}$ Ala substitutions may have a residual level of interaction with AC40 not detected by two-hybrid or coIP assay. Alternatively, additional protein-protein interactions, like those previously identified with other Pol III subunits, could contribute to the recruitment of Ty1 integration complex at Pol III transcribed-genes in the absence of the IN1-AC40 interaction (Cheung et al., 2016).

AC40 is common to both Pol I and Pol III, suggesting that IN1 may also interact with Pol I. Consistently, we found that Pol I is associated with IN1 in vivo. Pull-down of A190-TAP, the largest subunit of Pol I, retained WT IN1, whereas no association was detected with the IN1 mutants (Figure 3G). The RDN1 locus is composed of 100-200 tandem repeats of the 35Sprecursor $r D N A$, transcribed by Pol I, and the $5 \mathrm{~S} r D N A$, transcribed by Pol III (Dammann et al., 1993). Analysis of ChIP-seq reads mapping at multiple positions revealed WT HA-IN1 at this locus (Figure $3 \mathrm{H}$ ). IN1 occupancy may be overestimated, as reads corresponding to all repeats are aggregated on the two copies that are represented in the $S$. cerevisiae reference 
genome (https://www.yeastgenome.org). However, IN1 mutants compromising the IN1-AC40 interaction $-\mathrm{S}_{621} \mathrm{~A}, \mathrm{~L}_{622} \mathrm{~A}$, and particularly $\mathrm{K}_{617} \mathrm{~A}$ - reduced HA-IN1 occupancy at Pol Itranscribed loci. Thus, IN1, through its interaction with AC40, is also recruited to genes transcribed by Pol I.

Collectively, these results indicate that the IN1-AC40 interaction is necessary for the interaction between IN1 and Pol III and thus its recruitment at Pol III-transcribed genes. IN1 also interacts with Pol I via AC40 and is present at Pol I-transcribed genes.

\section{AC40 interaction defective Ty1 mutants have altered integration profiles.}

The reduced association of HA-IN1 mutants with Pol III-transcribed genes did not result in a significant increase in mutant HA-IN1 occupancy at other specific loci, as revealed by ChIPseq (Table S1). To investigate the integration profile of Ty1 mutants that have an impaired IN1-AC40 interaction, libraries of $\mathrm{His}^{+}$selected de novo Ty1 insertion events were generated in cells expressing WT or mutant Ty1his3AI elements from the GAL1 promoter (Barkova et al., 2018). We used an spt3-101 rad52 $\Delta$ mutant strain to avoid both trans-complementation of the mutant IN1 by endogenous WT IN1 and Rad52-dependent recombination events. Initially, we performed qualitative PCR to monitor Ty1 insertion events at the SUF16 tRNA gene and the SEO1 subtelomeric gene. These genes were identified as hot spots of Ty1 integration in WT and AC40sp loss-of-interaction mutant, respectively (Bridier-Nahmias et al., 2015). In independent cultures expressing WT Tylhis3AI, we observed multiple bands upstream of the SUF16 tRNA gene, characteristic of Ty1-HIS3 insertion in the three nucleosomes upstream of tDNA genes (Bachman et al., 2005; Dakshinamurthy et al., 2010) (Figure 4A). This profile was significantly different for Ty1his3 AI harboring $\mathrm{K}_{617} \mathrm{~A}, \mathrm{~S}_{621} \mathrm{~A}$, or $\mathrm{L}_{622} \mathrm{~A}$ mutations in IN1, with many fewer integration events upstream of SUF16, and increased insertion at SEO1, compared to WT Ty1his3AI (Figure 4A). This observation suggests that IN1 mutations at $\mathrm{K}_{617}, \mathrm{~S}_{621}$, and $\mathrm{L}_{622}$ have the same effect on Ty1 integration site targeting as the AC40sp lossof-interaction mutant.

To extend our analysis to the entire genome, we characterized Ty1-HIS3 de novo insertion event libraries using high-throughput sequencing. We could discriminate Ty1-HIS3 de novo insertions from endogenous elements using six nucleotides in the 3' LTR that were specific to the Tylhis3AI element (Baller et al., 2012). By comparing the Z-score of Ty1 insertions on 
four non-overlapping features (Figure 4B), we confirmed that WT Ty1 insertions occurred mainly in a 1-kb window upstream of most Pol III-transcribed genes (Table S2), the only exceptions being $t D N A s$ that were absent or not transcribed in our strain. There were significantly fewer Ty1-HIS3 insertions at Pol III-transcribed genes with the three IN1 mutants (Figure 4B). However, the preference for Pol III-transcribed genes was not completely lost, as insertions in these regions were higher than expected if Tyl targeting was random, supporting the idea that the interaction with Pol III is not fully abolished with these mutants.

WT Ty1-HIS3 insertions displayed a periodic profile in the region of the three nucleosomes located upstream of Pol III-transcribed genes, with two insertion sites per nucleosome, as seen previously (Figure 4C) (Baller et al., 2012; Bridier-Nahmias et al., 2015; Mularoni et al., 2012). This profile was modified with the three mutants, with the first site of the first nucleosome being less affected with the $\mathrm{S}_{621} \mathrm{~A}$ mutant, suggesting that close proximity to the $t D N A$ is a determinant of integration. The $\mathrm{K}_{617} \mathrm{~A}$ mutant, which had the lowest HA-IN1 occupancy at Pol III-transcribed genes (Figure 3D and 3E), displayed the largest decrease in integration events at Pol III-transcribed genes (Figures 4B and 4C). This correlation suggests that the strength of the IN1-AC40 interaction influences integration efficiency at Pol IIItranscribed genes. Concomitant with the decrease in integration at Pol III-transcribed genes, the three IN1 mutants showed an increase in integration events at the end of each chromosome arm, a phenotype that was most pronounced for the $\mathrm{K}_{617} \mathrm{~A}$ mutant (Figure 4D). These results are consistent with the redistribution of Ty1 insertions in these regions observed in the AC40sp loss-of-interaction mutant (Bridier-Nahmias et al., 2015). Ty1 de novo insertions at the ends of chromosomes were mostly located in regions defined as subtelomeres, based on heterochromatin specificities or loss of synteny between different Saccharomyces strains (Hocher et al., 2018; Yue et al., 2017), suggesting that these subtelomeric regions harbor determinants allowing Ty1 targeting (Figure 4E).

Altogether, these results further support a major role for the IN1-AC40 interaction in Ty1 integration targeting at Pol III-transcribed genes. They also confirm that, when this interaction is compromised, Ty1 insertions are not random but principally occur in subtelomeres. 


\section{The IN1 bNLS targets Ty5 integration at Pol III-transcribed genes.}

To determine whether the IN1 bNLS sequence is sufficient to confer Tyl integration site preferences, we transferred the sequence into the Ty5 retrotransposon, which preferentially integrates into heterochromatin at yeast silent mating loci (HMR and $H M L)$ and near telomeres (Zou et al., 1996). Ty5 selectivity relies on an interaction between a hexapeptide (TD5, targeting domain of Ty5) in the C-terminus of IN5 and the heterochromatin protein Sir4 (Gai and Voytas, 1998; Xie et al., 2001). Exchange of TD5 for the IN1 bNLS in IN5, expressed in a two-hybrid vector, resulted in IN5 interacting with AC40, but not with Sir4 (Figure 5A). WT IN5, IN5 lacking TD5 (IN5 $5_{\Delta \mathrm{TD} 5}$ ), and $\mathrm{IN} 5_{\Delta \mathrm{TD} 5+\mathrm{bNLS}}$ harboring the $\mathrm{L}_{622} \mathrm{~A}$ mutation in the bNLS sequence, all failed to interact with AC40, demonstrating that the interaction was strictly dependent on the IN1 bNLS sequence.

To establish whether the interaction with AC40 is sufficient to target IN5 $5_{\Delta \mathrm{TD} 5+\mathrm{bNLS}}$ to Pol IIItranscribed genes, we performed ChIP-seq in strains ectopically expressing HA-tagged IN5, $\mathrm{IN} 5_{\Delta \mathrm{TD} 5}$, and $\mathrm{IN} 5_{\Delta \mathrm{TD} 5+\mathrm{bNLS}}$. Metagene analysis of the tagged proteins binding to $t D N A \mathrm{~s}$ revealed a clear enrichment of IN $5_{\Delta \mathrm{TD} 5+\mathrm{bNLS}}$ at these loci, not detected for IN5 and IN $5_{\Delta \mathrm{TD} 5}$ (Figure 5B). IN5 $5_{\Delta \mathrm{TD} 5+\mathrm{bNLS}}$ was also present at the other Pol III-transcribed and Pol Itranscribed genes (Figure $5 \mathrm{C}$ and $\mathrm{S} 3 \mathrm{E}$ ). Overall, IN5 $5_{\triangle \mathrm{TD} 5+\mathrm{bNLS}}$ genome occupancy profile was very similar to that of IN1 (Pearson correlation of $\mathrm{R}=0.9$ Figure S3F and Table S3), confirming that the IN1 bNLS-AC40 interaction is sufficient for recruitment at Pol IIItranscribed genes. We did not detect IN5 enrichment at subtelomeric regions bound by Sir4 (Zill et al., 2010), nor at $H M L$ and $H M R$, which are Ty5 integration sites, which may be due to the weak association between Ty5 integration sites and Sir4 occupancy (Baller et al., 2011) and reflect a loose and dynamic interaction between IN5 and Sir4 proteins difficult to detect by ChIP.

To explore the impact of the bNLS-TD5 exchange on Ty5 integration site selectivity, we introduced $\mathrm{IN} 5_{\triangle \mathrm{TD} 5+\mathrm{bNLS}}$ into a functional Ty5his $3 A I$ reporter expressed from the GALI promoter. This replacement caused a 10-fold decrease in the frequency of Ty5 retrotransposition but did not inactivate the element (Figure S5G). $\mathrm{His}^{+}$colonies represented bona fide integration events, as a similar colony frequency was observed in the absence of homologous recombination (Figure $\mathrm{S} 5 \mathrm{G}$ ). $\mathrm{His}^{+}$selected de novo insertion events were generated for WT and mutant Ty5his $3 A I$ and the insertion profiles were monitored at specific loci by qualitative PCR (Figure 5D). We used an spt3-101 strain that does not express endogenous Tyl elements, to avoid interference between insertions of endogenous Ty1 and 
the mutant Ty5 at Pol-III transcribed genes. The Tylhis3AI profile displayed multiple insertion events at the Pol III-transcribed SCRI gene and glycine $t D N A s$, whereas no insertions were seen at $H M L$ and $H M R$. The WT Ty5 profile displayed multiple insertion events at $H M L$ and $H M R$, whereas no insertions were recovered at SCRI and very few were seen at the glycine $t D N A \mathrm{~s}$. In contrast, Ty $5_{\Delta \mathrm{TD} 5+\mathrm{bNLS}}$ did not integrate at $H M L$ and $H M R$, whereas multiple insertion events were detected at the Pol III-reporter genes. The difference in Ty1 and Ty $5_{\Delta \mathrm{TD} 5+\mathrm{bNLS}}$ banding patterns at Pol III-transcribed genes is probably due to Ty1, but not Ty5, preferentially integrating into nucleosomes, indicating that the Ty1 preference for nucleosomes is not dependent on interaction with AC40.

Together, these data indicate that the IN1 bNLS sequence is sufficient to direct the integration of the Ty5 retrotransposon upstream of Pol III-transcribed genes. 


\section{DISCUSSION}

Here, we show that the Ty1 IN1 bNLS plays a critical role in the interaction with the Ty1 tethering factor AC40. We demonstrate that the interaction with AC40 orchestrates the selection of Ty1 integration sites in the genome and that the IN1 bNLS can function as an independent module that targets the integration of another related retrotransposon upstream of the typical Ty1 Pol III-transcribed target genes.

We provide several lines of evidence supporting a major role for AC40 in recruiting Ty1 to both Pol III and Pol I-transcribed genes. First, IN1 interacts directly with AC40 in the absence of other yeast proteins. Second, mutations in the IN1 bNLS that reduce the interaction with AC40 abolish IN1 association with both Pol III and Pol I transcription complexes. Third, there is a concomitant decrease in IN1 occupancy and Ty1 integration at Pol I and Pol IIItranscribed loci when the IN1-AC40 is disrupted. Direct interactions were previously observed in vitro between IN1 and the C31, C34, and C53 Pol III-specific subunits (Cheung et al., 2016) but their precise role in the recruitment of the IN1 complex in vivo was not determined. The redistribution of Ty1 integration into subtelomeres, seen in the absence of the IN1-AC40 interaction ((Bridier-Nahmias et al., 2015) and this study) was not observed in a $\operatorname{rpc} 53 \triangle 2-280$ mutant, which decreases Ty1 integration at the SUF16 tRNA gene (Cheung et al., 2016). This suggests that either C53 secures IN1 binding to Pol III once IN1 has been recruited by AC40 or helps Ty1 integration at a step downstream of IN1 recruitment. Further studies will be necessary to address the role of the Pol III complex, and especially of C31, C34 and C53, in Ty1 integration.

We show that IN1 is present across Pol I-transcribed genes, and this presence is reduced by mutations that disrupt the IN1-AC40 interaction. Previous genome-wide mapping of Ty1 insertion sites did not reveal a clear pattern of Ty1 insertion into Pol I-transcribed genes at the RDN1 locus (Baller et al., 2012; Bridier-Nahmias et al., 2015; Mularoni et al., 2012), likely due to the highly repetitive nature of the $r D N A$ repeats (Bridier-Nahmias et al., 2015). Tyl insertion events at these loci seem to be rare (Bryk et al., 1997). This could be due in part to not all the repeats being transcribed by Pol I (Dammann et al., 1993) or to the rapid elimination of de novo Ty1 insertions by homologous recombination between RDN1 repeats. Alternatively, recruitment of IN1 might not be sufficient for Ty1 integration because the process requires additional cofactors or a chromatin structure that is only present at Pol IIItranscribed genes. 
Our IN1 mutants that disrupt the interaction with AC40 induce the same redistribution of Ty1 insertions at chromosome ends as seen in a AC40sp loss-of-interaction mutant (BridierNahmias et al., 2015). The insertion sites of these Tyl mutants are scattered throughout subtelomeric regions. Ty5 insertions also occur throughout subtelomeric regions (Baller et al., 2011). This scattered dispersion may explain why we have failed to detect IN1 and IN5 by ChIP-seq in these regions. High-resolution mapping of DNA binding sites will be required to address this point (Hafner et al., 2018; Meers et al., 2019).

To date, the specific retargeting of transposon integration sites has only been observed for Ty1. When the interaction between HIV, MLV, and Ty5 INs and their primary tethering factors (LEDGF/p75, BET proteins, and Sir4, respectively) is altered, the integration of these retroelements at their usual targets decreases substantially, and becomes random (Gai and Voytas, 1998; De Rijck et al., 2013; Schrijvers et al., 2012; Sharma et al., 2013; Wang et al., 2012). Chromosome ends are preferential targets of several TE families in different organisms (Casacuberta, 2017). In S. cerevisiae, subtelomeres are devoid of essential genes, are rich in stress responsive genes, and evolve rapidly in response to stress (Snoek et al., 2014) or domestication (Yue et al., 2017). Targeting of Ty1 integration may have evolved to provide a balance between integration into "safe" genomic regions, i.e. $t D N A s$, and integration into fast evolving regions when adaptation is necessary, i.e. subtelomeres. Accordingly, we propose that the IN1-AC40 interaction may be regulated by environmental stress. The observations that Ty5 targeted integration requires phosphorylation of the IN5 targeting domain, which is reduced by stress (Dai et al., 2007), and nutrient starvation regulates the Ty1 replication cycle (Morillon et al., 2000; Todeschini et al., 2005)) both lend support this hypothesis.

The pattern of integration of the Ty1 IN1 mutants largely overlaps in subtelomeric domains (Hocher et al., 2018; Yue et al., 2017). This correlation suggests that specific feature(s) in subtelomeres attract or facilitate Ty1 integration in the absence of the IN1-AC40 interaction. As we failed to detect an interaction between IN1 and Sir4, Ty1 integration into subtelomeres probably involves a mechanism different from that of Ty5. In a cis-targeting model, IN1 is tethered to subtelomeres through a weak interaction with a subtelomeric specific co-factor, such that the subtelomeric interaction will only be favored when binding to AC40 is compromised. The dispersed nature of Ty1 insertion sites suggests that the co-factor could be distributed across subtelomeres, like a histone mark specifically enriched in these regions (Hocher et al., 2018). Alternatively, IN1 co-factor could be present at a limited number of subtelomeric sites, and after recruitment, the Ty1 PIC would scan for a chromatin 
environment favorable for integration. Given Ty1 targets stable nucleosomes upstream of Pol III-transcribed genes (Baller et al., 2012; Mularoni et al., 2012), nucleosome stability could also be a determinant for integration at subtelomeres. A similar two-step targeting model has been proposed to explain the absence of correlation between Sir4 binding sites and Ty5 integration sites in subtelomeres (Baller et al., 2011). In a trans-targeting model, the proximity of the subtelomeres with the nuclear pores (Zimmer and Fabre, 2011), through which the Ty1 PIC transits, would facilitate Ty1 integration in these regions, especially when the interaction with AC40 is compromised. Consistent with this hypothesis, mutations in several components of the nuclear pore alter Tyl integration preferences (Manhas et al., 2018). HIV integration also occurs preferentially in chromatin proximal to the nuclear periphery (Lelek et al., 2015; Marini et al., 2015; Di Primio et al., 2013).

This work demonstrates that the IN1 bNLS functions as an independent module to target integration at Pol III-transcribed genes: The addition of this sequence to Ty5 IN is sufficient to direct Ty5 integration to these loci. Entry of the Ty1 PIC into the nucleus involves the classical import machinery and requires an interaction between importin- $\alpha$ and the IN1 bNLS (McLane et al., 2008). The IN1 NLS consists of two regions of basic amino acids, which are essential for IN1 nuclear import, separated by a linker sequence that also contributes, although to a lesser extent, to import (Kenna et al., 1998; Lange et al., 2011; Moore et al., 1998). The residues that are required for IN1 interaction with AC40 and Ty1 integration upstream of Pol III-transcribed genes cluster in a short peptide within the linker. Mutations in the linker substantially reduce the interaction with AC40 but not Ty1 retrotransposition frequency or IN1 nuclear import (this study). Thus, the IN1 bNLS is involved in nuclear import and integration targeting.

The IN1 linker has been proposed to induce a conformation that facilitates interaction of the two basic amino acid-rich regions with two NLS-binding pockets present in importin- $\alpha$ (Kosugi et al., 2009; Lange et al., 2011). The conformation of the linker when the basic amino acid-rich regions are bound to importin- $\alpha$ could expose the sequence recognized by AC40. Once in the nucleus, interaction with AC40 would assist Ran-GTP to dissociate the IN1importin- $\alpha$ complex (Passos et al., 2017; Rothenbusch et al., 2012), with nuclear entry and interaction with Pol III being coupled (Figure 6). Such coupling could promote Ty1 targeting to $t D N A \mathrm{~s}$, as these genes are recruited to the nuclear pore to be transcribed (Chen and Gartenberg, 2014).

Retroviral vectors have been used in gene therapy to correct various monogenic disorders. 
However, these vectors rely on the properties of HIV and MLV integrases, whose preferences for either transcribed genes (i.e. HIV) or promoters (i.e. MLV) make them potentially harmful for the genome (Anguela and High, 2019; Goswami et al., 2019). As Ty1 integration upstream of Pol III-transcribed genes preserves gene integrity (Bolton and Boeke, 2003), and given Pol III transcription and structure, including the presence of AC40, are highly conserved between yeast and humans, adapting Tyl-integration targeting to retroviral integrases could overcome the risk of insertional mutagenesis associated with current MLV and HIV retroviral-based vectors, allowing the development of safer retrovirus-based vectors for use in human gene transfer technologies. 
Acknowledgments. We thank A. Corbett and D. Voytas for plasmids; A. Bridier-Nahmias and members of the laboratory for stimulating discussions; A. Leseur for contribution during her training; E. Fabre, C. Fernandez-Tornero, G. Herrada, B. Palancade, J. Reguera for critical reading of the manuscript. This work was supported by intramural funding from Centre National de la Recherche Scientifique (CNRS), the Université Paris Diderot and the Institut National de la Santé et de la Recherche Médicale (INSERM), and from grants from the Fondation ARC pour la Recherche sur le Cancer (PJA 20151203412), the Agence Nationale de la Recherche through the generic call projects ANR-13-BSV3-0012 and ANR-17-CE110025. A.A-L was supported by a post-doctoral fellowship from Fondation pour la Recherche Médicale (FRM-SPF20170938755), A. B. by a post-doctoral fellowship from the ANR through the initiatives d'excellence (Idex ANR-11-IDEX-0005-02) and the Labex "Who am I?" (ANR11-LABX-0071) and I. A. by the PhD program from the CEA. This work has benefited from the facilities and expertise of the high-throughput sequencing platform of I2BC.

Supplementary Materials. Methods, Supplementary Tables S1-S6, Supplementary Figures S1-S3.

Author contributions. AAL, AB, CC, CG, HF, IA NP and RM performed the experiments. AAL performed computational analysis. AAL, AB, CC, JA and PL analyzed data and prepared figures AAL, AB, JA and PL wrote the manuscript. JA and PL conceived and supervised the study and secured funding.

Declaration of interests. The authors declare no competing interest. 


\section{FIGURE LEGENDS}

\section{Figure 1. bNLS linker sequence mutations abolish the interaction with AC40.}

A. Top. The Ty1 integrase (IN1) showing N-terminal and catalytic core domains (NTD and CCD) and the bipartite NLS at the C-terminus (CTD). Bottom. Alignment of amino acid sequences of Ty1, Ty2, and Ty4 integrase C-termini (IN1, IN2, and IN4, respectively). In bold: basic amino acids required for NLS function. In red: amino acids in the NLS linker relatively conserved between the three integrases. *, Identity; :, high similarity; ., low similarity; -, gap in sequence.

B. Two-hybrid interaction between GBD-AC40 and WT or mutant GAD-IN1 $1_{578-635}$. Alanine substitutions in $\mathrm{IN}_{578-635}$ are indicated. Cells were plated in two-fold serial dilutions on DOLeu-Trp-His plates to detect interaction. No growth or protein expression defects were detected (Figure S1A-B). +, Interaction; -, no interaction.

C. Two-hybrid interaction between GBD-AC40 and different IN1 regions fused to GAD, as indicated. Cells were plated in ten-fold serial dilutions on DO-Leu-Trp-His plates to detect interaction. No growth or protein expression defects were detected (Figure S1C-D). +, Interaction; -, no interaction.

D. In vitro interaction between $\mathrm{AC} 40$ and IN1 proteins co-expressed in E. coli. Immunoprecipitation of protein extracts was performed from bacteria cells expressing IN1-EPEA or $\mathrm{K}_{617} \mathrm{~A}, \mathrm{~S}_{621} \mathrm{~A}$, or $\mathrm{L}_{622} \mathrm{~A}$ IN1-EPEA mutants alone (-) or together with AC40-Twin-Strep-tag $(+)$. Cell lysates (Input) and immunoprecipitates (IP Strep) were analyzed by Western blot with anti-Strep-Tactin antibody and CaptureSelect ${ }^{\mathrm{TM}}$ Biotin Anti-C-tag Expected sizes are 41 $\mathrm{kDa}$ for AC40-Twin-Strep-tag and $100 \mathrm{kDa}$ for IN1-EPEA (WT and mutants).

\section{Figure 2. Non-AC40 binding IN1 mutants do not affect Ty1 integration frequency.}

A. Localization of $\mathrm{GFP}_{2}$-IN1 bNLS variants. Yeast cells expressing $\mathrm{GFP}_{2}$-IN1 bNLS variants and Nup49-mCherry were analyzed by direct fluorescence microscopy. The C-terminal 54 amino acids of IN1 containing the bNLS were fused to the C-terminus of two tandem GFPs $\left(\mathrm{GFP}_{2}\right.$-IN1 bNLS) to create a reporter protein that would be too large for passive diffusion

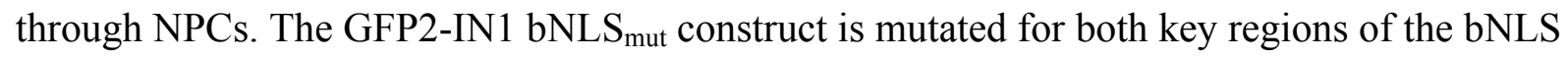


( ${ }_{596} \mathrm{KKR}_{598}$-AAA and ${ }_{628} \mathrm{KKR}_{630}$-AAA). Nup49-mCherry signal was used to visualize the location of the nuclear envelope. Corresponding DIC images are shown.

B. Retrotransposition frequency (log scale) of pGAL1-Ty1his3AI bearing substitutions of conserved residues by alanine $\left(\mathrm{K}_{617} \mathrm{~A}, \mathrm{M}_{619} \mathrm{~A}, \mathrm{R}_{620} \mathrm{~A}, \mathrm{~S}_{621} \mathrm{~A}\right.$, and $\left.\mathrm{L}_{622} \mathrm{~A}\right)$ in a spt3-101 rad52A strain. IN1 catalytic core domain mutant $\mathrm{D}_{154} \mathrm{~A}$ is defective for integration and NLS mutant $\left(\mathrm{KKR}_{628-630} \mathrm{GGT}\right)$ for nuclear import. Values are an average of four experiments, each performed with four independent colonies. ${ }^{*} \mathrm{p} \leq 0.05, * \mathrm{*} \leq 0.01$, and $* * * p \leq 0.001$; Student t-test.

\section{Figure 3. AC40 recruits IN1 at Pol I and Pol III-transcribed genes.}

A. Co-immunoprecipitation between endogenous Pol III (C160-HA) and ectopic IN1 tagged with streptavidin (IN1-Strep) expressed from the GAL1 promoter. Protein extracts of yeast cells expressing HA-tagged (+) or untagged (-) C160 respectively, and WT or $\mathrm{K}_{617} \mathrm{~A}, \mathrm{~S}_{621} \mathrm{~A}$, or $\mathrm{L}_{622} \mathrm{~A}$ IN1 mutants were incubated with anti-HA coated beads. Cell lysates (Input) and immunoprecipitates (IP HA) were analysed by immunoblotting with anti-HA and anti-Strep antibodies. Expected sizes are 160kDA for C160-HA and 100kDa for IN1-strep (WT and mutants).

B. Quantitative ChIP analysis of HA-IN1 enrichment at Pol III-transcribed genes. Immunoprecipitated DNA from yeast cells producing ectopic IN1 is expressed as a value relative to that of the input. Pol III transcribed-genes: $t D N A$-Leu and $t D N A$-Ile families (22 and 16 genes, respectively) and the unique SCR1 gene. GAL1 ORF serves as a control. The mean values and standard deviation (indicated by error bars) of at least three independent experiments are shown.

C. Fold enrichment of WT HA-IN1 over input at all the genes where HA-IN1 has been detected by ChIP-seq analysis. Each dot represents a gene. Grey, $t D N A \mathrm{~s}$; Red, other Pol IIItranscribed genes ; Blue, Pol II-transcribed genes.

D. WT and mutant IN1 association with all Pol III-transcribed genes (tRNA and ncRNA genes). Values obtained from ChIP seq analysis have been normalized in log2 RPKM (reads per kilobase per million mapped reads). Control is anti-HA immunoprecipitation of chromatin extracts in cells expressing IN1-Strep. 
E. Genome-wide occupancy profiles (top) and heatmaps (bottom) of WT and HA-IN1 mutants over a $1 \mathrm{~kb}$ window upstream and downstream of all $t D N A \mathrm{~s}$. Average ChIP-seq signals have been computed per 10bp bin, normalized to input and adjusted in log2 RPKM. Pearson correlation values corresponding to Figure S2B are indicated.

F. WT and mutant IN1 association to all Pol-II transcribed genes respectively. Values obtained from ChIP seq analysis have been normalized in log2 RPKM. Control, as described for panel D.

G. Co-immunoprecipitation between endogenous RNA Pol I (TAP-tagged-A190) and ectopic Ty1 integrase (IN1-HBH). Protein extracts of yeast cells expressing TAP-tagged $(+)$ or untagged (-) A190 and transformed with pCM185 derivatives expressing WT or $\mathrm{K}_{617} \mathrm{~A}$, $\mathrm{S}_{621} \mathrm{~A}$, or $\mathrm{L}_{622} \mathrm{~A}$ IN1-HBH mutants were incubated with $\mathrm{IgG}$ beads. Cell lysates (Input) and immunoprecipitates (IP TAP) were revealed by immunoblotting with anti-TAP and anti-Strep (five thousand-fold dilution). Expected sizes are 204 kDA for A190-HA and $82 \mathrm{kDa}$ for IN1strep (WT and mutants).

H. Genome browser visualization of HA-IN1 occupancy at the RDN1 locus encoding ribosomal RNA genes. The 100 to $200 R D N 1$ repeats of the yeast genome on chromosome $\mathrm{XII}$ are aggregated into two repeats. $R D N 37-1$ and $R D N 37-2$ are transcribed by RNA Polymerase I as a 35S precursor rRNA. RDN5-1 and RDN5-2 are transcribed by RNA Polymerase III to give the 5S RNA. Occupancy of WT HA-IN1 and $\mathrm{K}_{617} \mathrm{~A}, \mathrm{~S}_{621} \mathrm{~A}$ and $\mathrm{L}_{622} \mathrm{~A}$ HA-IN1 mutants is represented in each panel. Control, as described for panel D. Values obtained from ChIP seq analysis have been normalized for each condition (WT and mutant IN1) to input and adjusted in $\log 2$ RPKM.

\section{Figure 4. AC40 interaction defective Ty1 mutants have altered integration profiles.}

A. Detection of de novo Ty1 insertions upstream of the SUF16 and SEO1 genes by PCR using a primer in HIS3 (red triangle) and a primer in the locus of interest (blue triangle). Ty1 retrotransposition was induced in spt3-101 rad524 cells transformed with plasmids expressing WT or mutant (IN1 $\mathrm{K}_{617} \mathrm{~A}, \mathrm{~S}_{621} \mathrm{~A}$ and $\mathrm{L}_{622} \mathrm{~A}$ ) Ty1his $3 A I$ from the GAL1 promoter. Total genomic DNA was extracted from $\mathrm{His}^{+}$cells obtained from independent cultures induced for retrotransposition. 
B. Genome-Wide Ty1 insertion frequencies at each genomic feature are clustered in a heatmap. Score is computed in column Z-score. ORFs, all RNA Pol II transcribed genes except gene at subtelomeres; rDNA, one RDN1 copy; up 1kb Pol III, $1 \mathrm{~kb}$ upstream of all Pol III transcribed genes; Subtelomeres, genomic coordinates corresponding to chromatin covered by Sir2 and Sir3, when they are co-overexpressed (Hocher et al., 2018); Random, 100.000 random Ty1 computed insertions in the genome.

C. Ty1 insertion profile upstream of $t D N A \mathrm{~s}$. Total genomic DNA extracted in (B) was prepared for Ty1 de novo integration event sequencing. Ty1 insertions are computed in a $1 \mathrm{~kb}$ window upstream of all the 275 nuclear $t D N A$ s (position 0 in the graph). Each position is divided by the number of insertions at this position (weight). The Smoothing curves indicate the general trend. Nucleosome center positions are from (Brogaard et al., 2012).

D. Ty1 insertion frequencies for each left and right subtelomere of chromosomes are clustered in a heatmap. Score is computed in row Z-score. Random, as described for panel B.

E. Genome browser visualization of WT and IN1 $\mathrm{K}_{617} \mathrm{~A}$ mutant Ty1-HIS3 insertions into chromosome V left and chromosome VI right subtelomeres compare to the subtelomere boundaries defined by (Hocher et al., 2018; Yue et al., 2017). Red stars indicate the first essential gene of each subtelomere.

\section{Figure 5. The IN1 bNLS targets Ty5 integration at Pol III-transcribed genes.}

A. Two-hybrid interaction between GBD-Sir4 (left) or GBD-AC40 (right) and different GAD-IN5 or GAD-IN1 constructions. $\mathrm{IN} 1_{578-635}$ and $\mathrm{IN} 1_{1-578}$ are used as positive and negative control for interaction with AC40, respectively. IN5 and IN5 $5_{\Delta \mathrm{TD} 5}$ are used as positive and negative control for interaction with Sir4, respectively. Cells were plated in five-fold serial dilutions on DO-Leu-Trp-His plates to detect interaction. No growth or protein expression defects were detected (Figure S5A-B).

B. Genome-wide occupancy profiles (top) and heatmaps (bottom) of IN5, WT, and indicated mutants, $+/-1 \mathrm{~kb}$ upstream and downstream of all $t D N A \mathrm{~s}$. Average ChIP-seq signals have been computed per 10bp bin, normalized to the input and adjusted in RPKM.

C. Genome browser visualization of different HA-IN occupancy for chromosome V (chrV:431129..443275). Occupancy of WT IN5, IN5 $5_{\Delta \mathrm{TD}}, \mathrm{IN} 5_{\Delta \mathrm{TD}+\mathrm{bNLS}}$ and WT IN1 is 
represented in each panel. Control, anti-HA immunoprecipitation of chromatin extracts expressing IN1-Strep. The region contains four tDNAs (tH(GUG)E2, tK(CUU)E2, tV(ACC)E1, tI(AAU)E1) and two ncRNA genes transcribed by Pol III (SNR52 and SCRl). Values obtained from ChIP seq analysis have been normalized to input and adjusted in RPKM.

D. Detection by PCR of Ty5, Ty $5_{\Delta \mathrm{TD} 5+\mathrm{bNLS}}$ or Ty1 de novo integration events at the $H M R$ and $H M L$ loci, SCR 1 and upstream of all glycine $t D N A$ s using a primer in HIS3 and a primer in the locus of interest. Retrotransposition was induced for three to four days at $20^{\circ} \mathrm{C}$ in spt3-101 cells transformed with WT or mutated pGAL1-Ty5his $3 A I$ (Ty5 or Ty $5_{\triangle \mathrm{TD} 5+\mathrm{bNLS}}$ ) and pGAL1Ty1his3AI (Ty1). Genomic DNA was extracted from HIS $^{+}$cells for subsequent PCR assays. Ty5, Ty $5_{\Delta \mathrm{TD} 5+\mathrm{bNLS}}$ or Ty1 de novo integration events were detected by using a primer in HIS3 gene and a primer in the gene of interest. PCR have been performed on genomic DNA extracted from three independent retrotransposition assays.

Figure 6. Model coupling the nuclear entry of Ty1 pre-integration complex with Ty1 integration at Pol III-transcribed genes.

1) The Ty1 IN1 bNLS binds to importin- $\alpha$, allowing the Tyl pre-integration complex to enter the nucleus. 2) Pol III genes are transcribed at the nuclear pores (Chen and Gartenberg, 2014). The conformation adopted by the IN1 bNLS linker sequence when the two basic amino acidrich regions are bound to importin- $\alpha$ exposes the sequence recognized by AC40. 3) The binding of AC40 to IN1 assists Ran-GTP dissociate of the IN1-importin- $\alpha$ complex. 4) The association with AC40, in the Pol III complex, targets Ty1 integration upstream of Pol IIItranscribed genes. 


\section{REFERENCES}

Anguela, X.M., and High, K.A. (2019). Entering the Modern Era of Gene Therapy. Annu. Rev. Med. 70, 273-288.

Bachman, N., Gelbart, M.E., Tsukiyama, T., and Boeke, J.D. (2005). TFIIIB subunit Bdp1p is required for periodic integration of the Ty1 retrotransposon and targeting of Isw2p to $\mathrm{S}$. cerevisiae tDNAs. Genes Dev 19, 955-964.

Baller, J.A., Gao, J., and Voytas, D.F. (2011). Access to DNA establishes a secondary target site bias for the yeast retrotransposon Ty5. Proc Natl Acad Sci U S A 108, 20351-20356.

Baller, J.A., Gao, J., Stamenova, R., Curcio, M.J., and Voytas, D.F. (2012). A nucleosomal surface defines an integration hotspot for the Saccharomyces cerevisiae Ty1 retrotransposon. Genome Res 22, 704-713.

Barkova, A., Asif-Laidin, A., and Lesage, P. (2018). Genome-Wide Mapping of Yeast Retrotransposon Integration Target Sites. In Methods in Enzymology (Elsevier Inc.) 612, 197-223.

Boeke, J.D., and Devine, S.E. (1998). Yeast retrotransposons: finding a nice quiet neighborhood. Cell 93, 1087-1089.

Bolton, E.C., and Boeke, J.D. (2003). Transcriptional interactions between yeast tRNA genes, flanking genes and Ty elements: a genomic point of view. Genome Res 13, 254-263.

Bourque, G. (2009). Transposable elements in gene regulation and in the evolution of vertebrate genomes. Curr. Opin. Genet. Dev. 19, 607-612.

Bridier-Nahmias, A., Tchalikian-Cosson, A., Baller, J.A., Menouni, R., Fayol, H., Flores, A., Saïb, A., Werner, M., Voytas, D., and Lesage, P. (2015). An RNA polymerase III subunit determines sites of retrotransposon integration. Science 348, 585-588.

Brogaard, K., Xi, L., Wang, J.P., and Widom, J. (2012). A map of nucleosome positions in yeast at base-pair resolution. Nature 486, 496-501.

Bryk, M., Banerjee, M., Murphy, M., Knudsen, K.E., Garfinkel, D.J., and Curcio, M.J. (1997). Transcriptional silencing of Ty1 elements in the RDN1 locus of yeast. Genes Dev. 11, 255-269.

Carr, M., Bensasson, D., and Bergman, C.M. (2012). Evolutionary Genomics of Transposable Elements in Saccharomyces cerevisiae. PLoS One 7, e50978.

Casacuberta, E. (2017). Drosophila: Retrotransposons making up telomeres. Viruses 9.

Chen, M., and Gartenberg, M.R. (2014). Coordination of tRNA transcription with export at nuclear pore complexes in budding yeast. Genes Dev. 28, 959-970.

Cherepanov, P., Maertens, G., Proost, P., Devreese, B., Van Beeumen, J., Engelborghs, Y., De Clercq, E., and Debyser, Z. (2003). HIV-1 integrase forms stable tetramers and associates with LEDGF/p75 protein in human cells. J. Biol. Chem. 278, 372-381.

Cheung, S., Ma, L., Chan, P.H.W., Hu, H.-L., Mayor, T., Chen, H.-T., and Measday, V. (2016). Ty1-Integrase interacts with RNA Polymerase III specific subcomplexes to promote insertion of Ty1 elements upstream of Pol III-transcribed genes. J. Biol. Chem. 296, 63916411.

Cheung, S., Manhas, S., and Measday, V. (2018). Retrotransposon targeting to RNA polymerase III-transcribed genes. Mob. DNA 9, 1-15. 
Chuong, E.B., Elde, N.C., and Feschotte, C. (2016). Regulatory activities of transposable elements: from conflicts to benefits. Nat Rev Genet 18, 71-86.

Cosby, R.L., Chang, N., and Feschotte, C. (2019). Host - transposon interactions : conflict, cooperation, and cooption. Genes Dev. 33, 1098-1116.

Curcio, M.J., and Garfinkel, D.J. (1991). Single-step selection for Ty1 element retrotransposition. Proc. Natl. Acad. Sci. U S A 88, 936-940.

Dai, J., Xie, W., Brady, T.L., Gao, J., and Voytas, D.F. (2007). Phosphorylation regulates integration of the yeast Ty5 retrotransposon into heterochromatin. Mol Cell 27, 289-299.

Dakshinamurthy, A., Nyswaner, K.M., Farabaugh, P.J., and Garfinkel, D.J. (2010). BUD22 affects Ty1 retrotransposition and ribosome biogenesis in Saccharomyces cerevisiae. Genetics 185, 1193-1205.

Dammann, R., Lucchini, R., Koller, T., and Sogo, J.M. (1993). Chromatin structures and transcription of rDNA in yeast Saccharomyces cerevisiae. Nucleic Acids Res. 21, 2331-2338.

Devine, S.E., and Boeke, J.D. (1996). Integration of the yeast retrotransposon Ty1 is targeted to regions upstream of genes transcribed by RNA polymerase III. Genes Dev. 10, 620-633.

Fujiwara, H., Osanai, M., Matsumoto, T., and Kojima, K.K. (2005). Telomere-specific nonLTR retrotransposons and telomere maintenance in the silkworm, Bombyx mori.

Chromosom. Res 13, 455-467.

Gai, X., and Voytas, D.F. (1998). A single amino acid change in the yeast retrotransposon Ty5 abolishes targeting to silent chromatin. Mol Cell 1, 1051-1055.

Goswami, R., Subramanian, G., Silayeva, L., Newkirk, I., Doctor, D., Chawla, K., Chattopadhyay, S., Chandra, D., Chilukuri, N., and Betapudi, V. (2019). Gene therapy leaves a vicious cycle. Front. Oncol. 9, 1-25.

Guo, Y., and Levin, H.L. (2010). High-throughput sequencing of retrotransposon integration provides a saturated profile of target activity in Schizosaccharomyces pombe. Genome Res. 20, 239-248.

Gupta, S.S., Maetzig, T., Maertens, G.N., Sharif, A., Rothe, M., Weidner-Glunde, M., Galla, M., Schambach, A., Cherepanov, P., and Schulz, T.F. (2013). Bromo- and extraterminal domain chromatin regulators serve as cofactors for murine leukemia virus integration. J. Virol. 87, 12721-12736.

Hafner, L., Lezaja, A., Zhang, X., Lemmens, L., Shyian, M., Albert, B., Follonier, C., Nunes, J.M., Lopes, M., Shore, D., et al. (2018). Rif1 Binding and Control of Chromosome-Internal DNA Replication Origins Is Limited by Telomere Sequestration. Cell Rep. 23, 983-992.

Hickey, a., Esnault, C., Majumdar, A., Chatterjee, a. G., Iben, J.R., McQueen, P.G., Yang, a. X., Mizuguchi, T., Grewal, S.I.S., and Levin, H.L. (2015). Single Nucleotide Specific Targeting of the Tf1 Retrotransposon Promoted by the DNA-Binding Protein Sap1 of Schizosaccharomyces pombe. Genetics 201, 905-924.

Hocher, A., Ruault, M., Kaferle, P., Descrimes, M., Garnier, M., Morillon, A., and Taddei, A. (2018). Expanding heterochromatin reveals discrete subtelomeric domains delimited by chromatin landscape transitions. Genome Res. 28, 1867-1881.

Huang, Q., Purzycka, K.J., Lusvarghi, S., Li, D., Legrice, S.F.J., and Boeke, J.D. (2013). Retrotransposon Ty1 RNA contains a 5'-terminal long-range pseudoknot required for efficient reverse transcription. RNA 19, 320-332. 
Jacobs, J.Z., Rosado-Lugo, J.D., Cranz-Mileva, S., Ciccaglione, K.M., Tournier, V., and Zaratiegui, M. (2015). Arrested replication forks guide retrotransposon integration. Science $349,1549-1553$.

Ji, H., Moore, D.P., Blomberg, M.A., Braiterman, L.T., Voytas, D.F., Natsoulis, G., and Boeke, J.D. (1993). Hotspots for unselected Tyl transposition events on yeast chromosome III are near tRNA genes and LTR sequences. Cell 73, 1007-1018.

Kenna, M.A., Brachmann, C.B., Devine, S.E., and Boeke, J.D. (1998). Invading the Yeast Nucleus: a Nuclear Localization Signal at the C Terminus of Ty1 Integrase Is Required for Transposition In Vivo. Mol. Cell. Biol. 18, 1115-1124.

Kim, J.M., Vanguri, S., Boeke, J.D., Gabriel, A., and Voytas, D.F. (1998). Transposable elements and genome organization: A comprehensive survey of retrotransposons revealed by the complete Saccharomyces cerevisiae genome sequence. Genome Res. 8, 464-478.

Kirchner, J., Connolly, C.M., and Sandmeyer, S.B. (1995). Requirement of RNA polymerase III transcription factors for in vitro position-specific integration of a retroviruslike element. Science 267, 1488-1491.

Kling, E., Spaller, T., Schiefner, J., Bönisch, D., and Winckler, T. (2018). Convergent evolution of integration site selection upstream of tRNA genes by yeast and amoeba retrotransposons. Nucleic Acids Res. 46, 7250-7260.

Kosugi, S., Hasebe, M., Matsumura, N., Takashima, H., Miyamoto-Sato, E., Tomita, M., and Yanagawa, H. (2009). Six classes of nuclear localization signals specific to different binding grooves of importin $\alpha$. J. Biol. Chem. 284, 478-485.

Kumar, Y., and Bhargava, P. (2013). A unique nucleosome arrangement, maintained actively by chromatin remodelers facilitates transcription of yeast tRNA genes. BMC Genomics 14, 402.

Lange, A., Mclane, L.M., Mills, R.E., Devine, S.E., and Corbett, A.H. (2011). Expanding the Definition of the Classical Bipartite Nuclear Localization Signal. Traffic 11, 311-323.

Lelek, M., Casartelli, N., Pellin, D., Rizzi, E., Souque, P., Severgnini, M., Di Serio, C., Fricke, T., Diaz-Griffero, F., Zimmer, C., et al. (2015). Chromatin organization at the nuclear pore favours HIV replication. Nat. Commun. 6, 6483.

Levin, H.L., and Moran, J. V (2011). Dynamic interactions between transposable elements and their hosts. Nat Rev Genet 12, 615-627.

Llano, M., Saenz, D.T., Meehan, A., Wongthida, P., Peretz, M., Walker, W.H., Teo, W., and Poeschla, E.M. (2006). An essential role for LEDGF/p75 in HIV integration. Science 314, 461-464.

Manhas, S., Ma, L., and Measday, V. (2018). The yeast Ty1 retrotransposon requires components of the nuclear pore complex for transcription and genomic integration. Nucleic Acids Res. 46, 3552-3578.

Marini, B., Kertesz-Farkas, A., Ali, H., Lucic, B., Lisek, K., Manganaro, L., Pongor, S., Luzzati, R., Recchia, A., Mavilio, F., et al. (2015). Nuclear architecture dictates HIV-1 integration site selection. Nature 521, 227-231.

McLane, L.M., Pulliam, K.F., Devine, S.E., and Corbett, A.H. (2008). The Ty1 integrase protein can exploit the classical nuclear protein import machinery for entry into the nucleus. Nucleic Acids Res. 36, 4317-4326.

Meers, M.P., Bryson, T.D., Henikoff, J.G., and Henikoff, S. (2019). Improved CUT\&RUN 
chromatin profiling tools. Elife 8, 1-16.

Moore, S.P., Rinckel, L.A., and Garfinkel, D.J. (1998). A Ty1 Integrase Nuclear Localization Signal Required for Retrotransposition. Mol Cell Biol 18, 1105-1114.

Moqtaderi, Z., and Struhl, K. (2004). Genome-Wide Occupancy Profile of the RNA Polymerase III Machinery in Saccharomyces cerevisiae Reveals Loci with Incomplete Transcription Complexes †. Mol. Cell. Biol. 24, 4118-4127.

Morillon, A., Springer, M., and Lesage, P. (2000). Activation of the Kss1 invasivefilamentous growth pathway induces Tyl transcription and retrotransposition in Saccharomyces cerevisiae. Mol Cell Biol 20, 5766-5776.

Mularoni, L., Zhou, Y., Bowen, T., Gangadharan, S., Wheelan, S.J., and Boeke, J.D. (2012). Retrotransposon Ty1 integration targets specifically positioned asymmetric nucleosomal DNA segments in tRNA hotspots. Genome Res. 22, 693-703.

Naito, K., Zhang, F., Tsukiyama, T., Saito, H., Hancock, C.N., Richardson, A.O., Okumoto, Y., Tanisaka, T., and Wessler, S.R. (2009). Unexpected consequences of a sudden and massive transposon amplification on rice gene expression. Nature 461, 1130-1134.

Oficjalska-Pham, D., Harismendy, O., Smagowicz, W.J., Gonzalez de Peredo, A., Boguta, M., Sentenac, A., and Lefebvre, O. (2006). General repression of RNA polymerase III transcription is triggered by protein phosphatase type 2A-mediated dephosphorylation of Maf1. Mol Cell 22, 623-632.

Pardue, M.-L.L., and DeBaryshe, P.G. (2011). Retrotransposons that maintain chromosome ends. Proc Natl Acad Sci U S A 108, 20317-20324.

Passos, D.O., Li, M., Yang, R., Rebensburg, S. V, Ghirlando, R., Jeon, Y., Shkriabai, N., Kvaratskhelia, M., Craigie, R., and Lyumkis, D. (2017). Cryo-EM structures and atomic model of the HIV-1 strand transfer complex intasome. Science 355, 89-92.

Patterson, K., Shavarebi, F., Magnan, C., Chang, I., Qi, X., Baldi, P., Bilanchone, V., and Sandmeyer, S.B. (2019). Local features determine Ty3 targeting frequency at RNA polymerase III transcription start sites. Genome Res. 29, 1298-1309.

Penton, E.H., and Crease, T.J. (2004). Evolution of the transposable element Pokey in the ribosomal DNA of species in the subgenus Daphnia (Crustacea: Cladocera). Mol. Biol. Evol. $21,1727-1739$.

Di Primio, C., Quercioli, V., Allouch, A., Gijsbers, R., Christ, F., Debyser, Z., Arosio, D., and Cereseto, A. (2013). Single-Cell Imaging of HIV-1 Provirus (SCIP). Proc. Natl. Acad. Sci. U. S. A. $110,5636-5641$.

De Rijck, J., de Kogel, C., Demeulemeester, J., Vets, S., El Ashkar, S., Malani, N., Bushman, F.D., Landuyt, B., Husson, S.J., Busschots, K., et al. (2013). The BET Family of Proteins Targets Moloney Murine Leukemia Virus Integration near Transcription Start Sites. Cell Rep. $5,886-894$.

Rothenbusch, U., Sawatzki, M., Chang, Y., Caesar, S., and Schlenstedt, G. (2012).

Sumoylation regulates Kap114-mediated nuclear transport. EMBO J. 31, 2461-2472.

Schrijvers, R., Vets, S., De Rijck, J., Malani, N., Bushman, F.D., Debyser, Z., and Gijsbers, R. (2012). HRP-2 determines HIV-1 integration site selection in LEDGF/p75 depleted cells. Retrovirology 9, 84.

Sharma, A., Larue, R.C., Plumb, M.R., Malani, N., Male, F., Slaughter, A., Kessl, J.J., Shkriabai, N., Coward, E., Aiyer, S.S., et al. (2013). BET proteins promote efficient murine 
leukemia virus integration at transcription start sites. Proc. Natl. Acad. Sci. U. S. A. 110, 12036-12041.

Sharon, G., Burkett, T.J., and Garfinkel, D.J. (1994). Efficient homologous recombination of Ty1 element cDNA when integration is blocked. Mol Cell Biol 14, 6540-51.

Snoek, T., Voordeckers, K., and Verstrepen, K.J. (2014). Chapter 3 : Subtelomeric regions promote evolutionary innovation of gene families in yeast. In Subtelomeres, E.J. Louis, and M.M. Becker, eds. (Springer), pp. 39-70.

Spaller, T., Kling, E., Glöckner, G., Hillmann, F., and Winckler, T. (2016). Convergent evolution of tRNA gene targeting preferences in compact genomes. Mob. DNA 7, 17.

Sultana, T., Zamborlini, A., Cristofari, G., and Lesage, P. (2017). Integration site selection by retroviruses and transposable elements in eukaryotes. Nat. Rev. Genet. 18, 292-308.

Teytelman, L., Thurtle, D.M., Rine, J., and Van Oudenaarden, A. (2013). Highly expressed loci are vulnerable to misleading ChIP localization of multiple unrelated proteins. Proc. Natl. Acad. Sci. U. S. A. 110, 18602-18607.

Todeschini, A.L., Morillon, A., Springer, M., and Lesage, P. (2005). Severe adenine starvation activates Tyl transcription and retrotransposition in Saccharomyces cerevisiae. Mol Cell Biol 25, 7459-7472.

Wang, H., Jurado, K. a, Wu, X., Shun, M.-C., Li, X., Ferris, A.L., Smith, S.J., Patel, P. a, Fuchs, J.R., Cherepanov, P., et al. (2012). HRP2 determines the efficiency and specificity of HIV-1 integration in LEDGF/p75 knockout cells but does not contribute to the antiviral activity of a potent LEDGF/p75-binding site integrase inhibitor. Nucleic Acids Res. 40, $11518-11530$.

Wilhelm, M., and Wilhelm, F.-X. (2005). Role of Integrase in Reverse Transcription of the Saccharomyces cerevisiae Retrotransposon Ty1. Eukaryot. Cell 4, 1057-1065.

Wilhelm, F.X., Wilhelm, M., and Gabriel, A. (2005). Reverse transcriptase and integrase of the Saccharomyces cerevisiae Ty1 element. Cytogenet Genome Res 110, 269-287.

Winston, F., Durbin, K.J., and Fink, G.R. (1984). The SPT3 gene is required for normal transcription of Ty elements in S. cerevisiae. Cell 39, 675-82.

Xie, W., Gai, X., Zhu, Y., Zappulla, D.C., Sternglanz, R., and Voytas, D.F. (2001). Targeting of the yeast Ty 5 retrotransposon to silent chromatin is mediated by interactions between integrase and Sir4p. Mol Cell Biol 21, 6606-6614.

Ye, J., Pérez-González, C.E., Eickbush, D.G., and Eickbush, T.H. (2005). Competition between R1 and R2 transposable elements in the 28S rRNA genes of insects. Cytogenet. Genome Res. 110, 299-306.

Yue, J.X., Li, J., Aigrain, L., Hallin, J., Persson, K., Oliver, K., Bergström, A., Coupland, P., Warringer, J., Lagomarsino, M.C., et al. (2017). Contrasting evolutionary genome dynamics between domesticated and wild yeasts. Nat. Genet. 49, 913-924.

Zill, O.A., Scannell, D., Teytelman, L., and Rine, J. (2010). Co-evolution of transcriptional silencing proteins and the DNA elements specifying their assembly. PLoS Biol. 8. e1000550.

Zimmer, C., and Fabre, E. (2011). Principles of chromosomal organization: lessons from yeast. J. Cell Biol. 192, 723-733.

Zou, S., Ke, N., Kim, J.M., and Voytas, D.F. (1996). The Saccharomyces Retrotransposon Ty5 Integrates Preferentially into Regions of Silent Chromatin at the Telomeres and Mating 
bioRxiv preprint doi: https://doi.org/10.1101/2019.12.18.879569; this version posted December 18, 2019. The copyright holder for this

preprint (which was not certified by peer review) is the author/funder, who has granted bioRxiv a license to display the preprint in perpetuity. It is made available under aCC-BY-NC-ND 4.0 International license.

Loci. Genes Dev 10, 634-645. 
Figure 1. bNLS linker sequence mutations abolish the interaction with AC40.

A

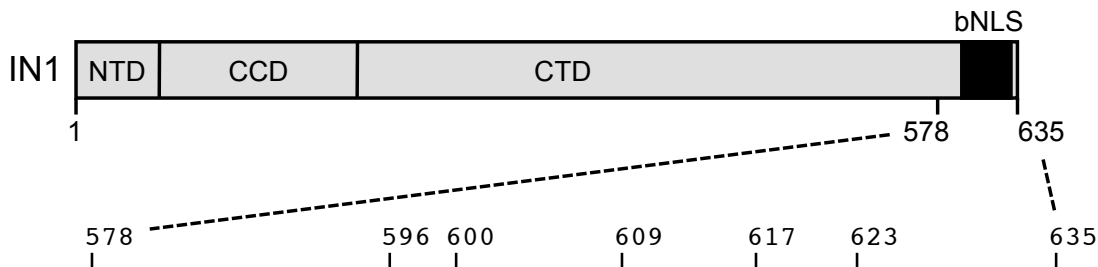

IN1 NSSLGGIGDSNAYTTINSKKRSLEDNE-TEIKVSRDTWNTKNMRSLEPPRSKKRIHLIA

IN2 NSSLGGMDDSNVLTTTKSKKRSLEDNE-TEIEVSRDTWNNKNMRSLEPPRSKKRINLIA

IN4 SPRL---------------EQNIEASGSPVQTVNKSAFLNKEFSSLNMKRKRKRKRKRKRHDKNNSLTSYELERDKKRSKRNRVKLIP * : : : * . *.: : .*: : * : : : :

B

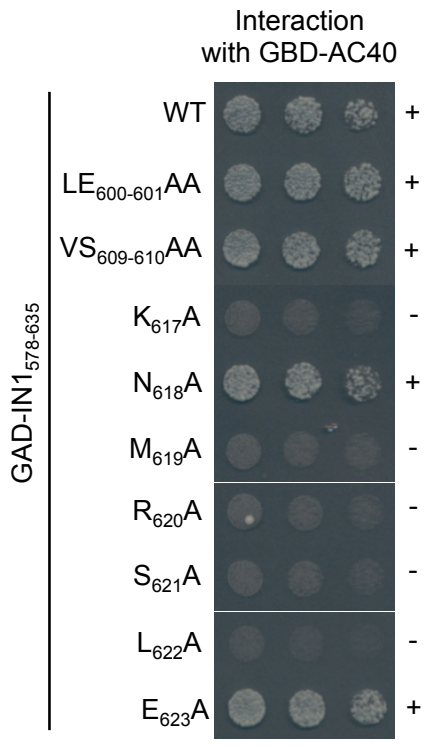

C

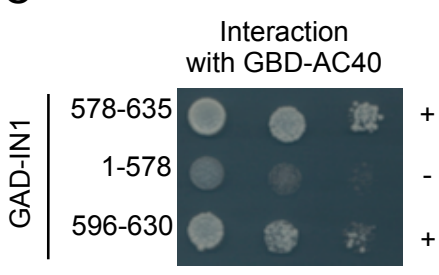

D

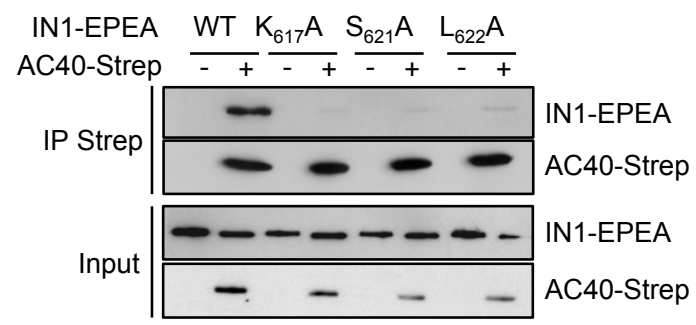


Figure 2. Non-AC40 binding IN1 mutants do not affect Ty1 integration frequency.

A

Nup49-

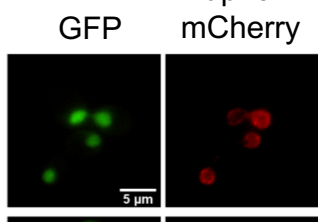

IN1 bNLS

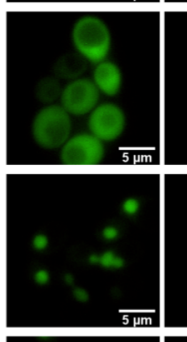

IN1 bNLS

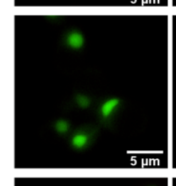

IN1 bNLS ${ }_{\text {L622A }}$
B

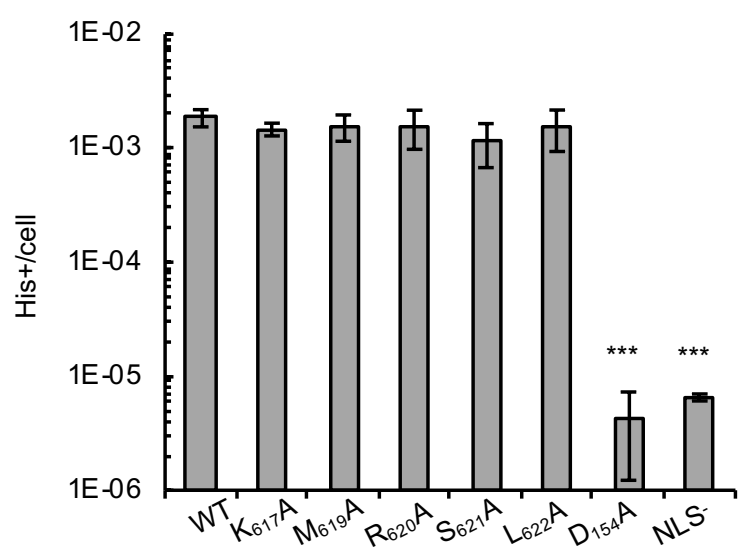


Figure 3. AC40 recruits IN1 at Pol I and Pol III-transcribed genes.

A

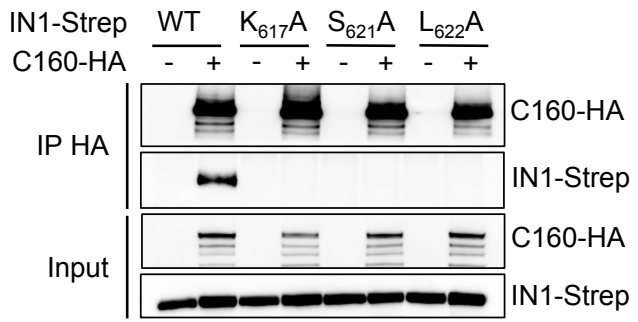

C

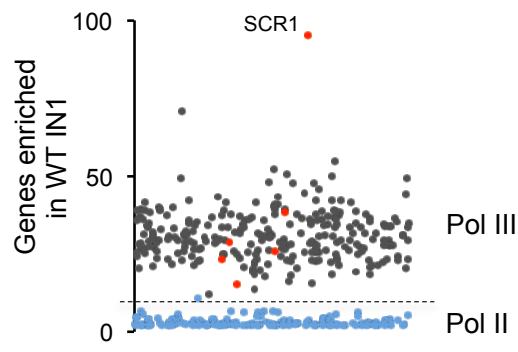

$E$

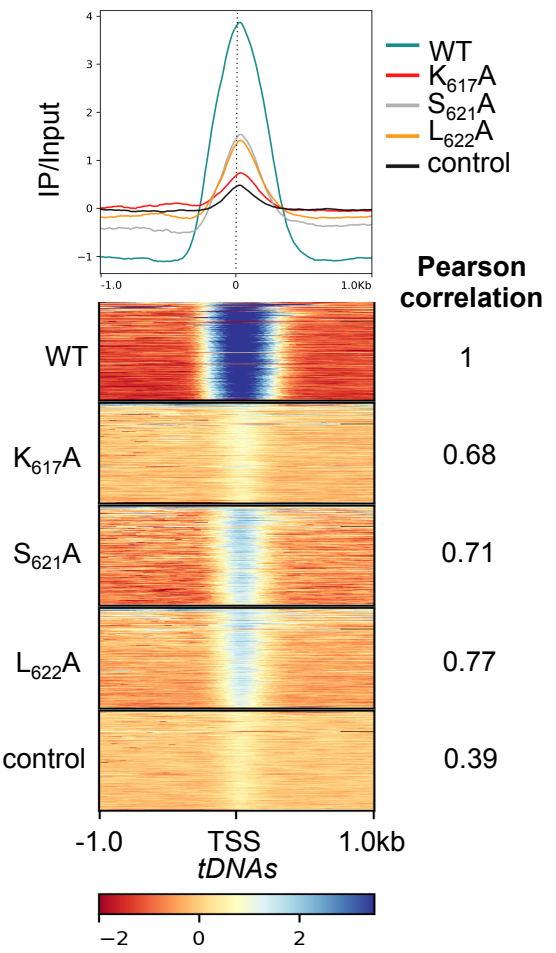

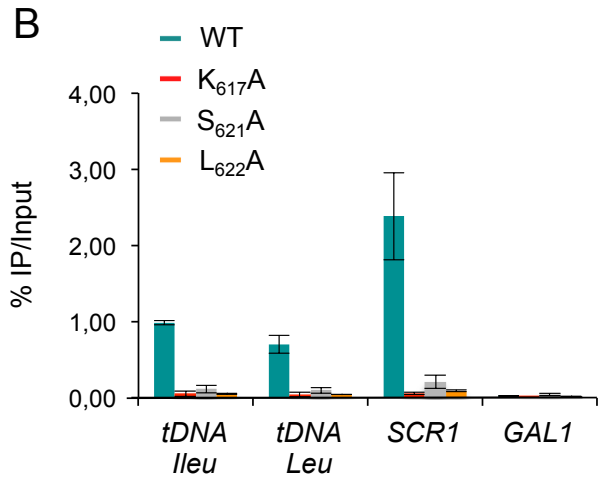

D

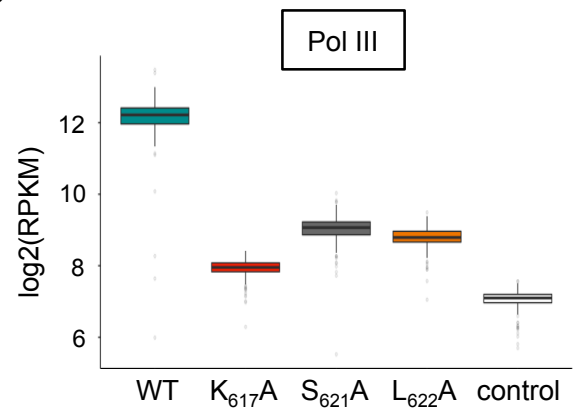

$\mathrm{F}$

\section{Pol II}

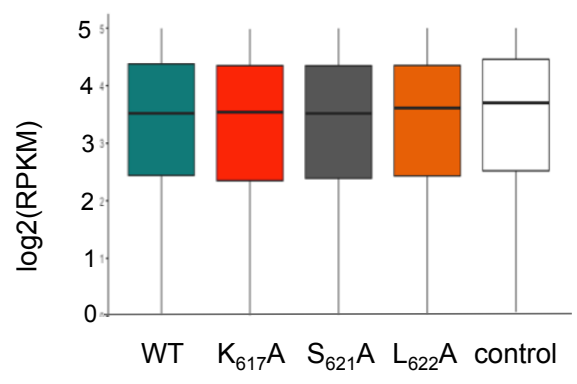

G

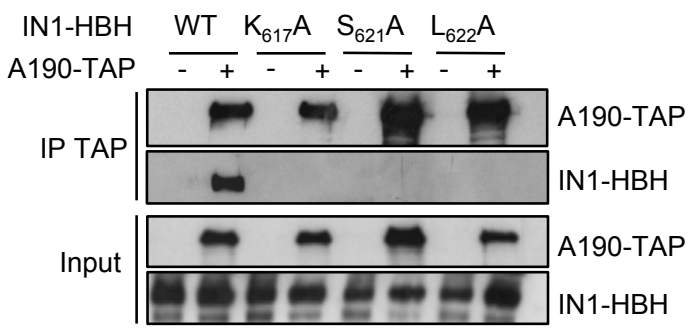

$\mathrm{H}$

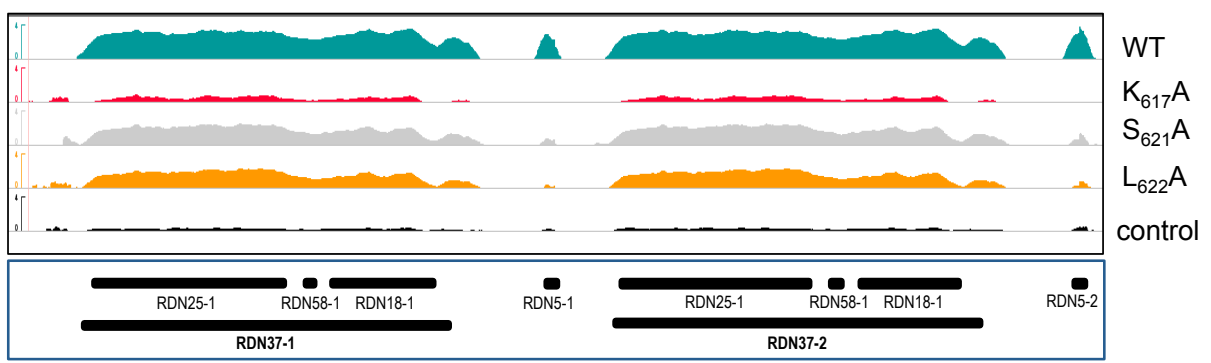


Figure 4. Non-AC40 binding Ty1 mutants have altered integration profiles.

A

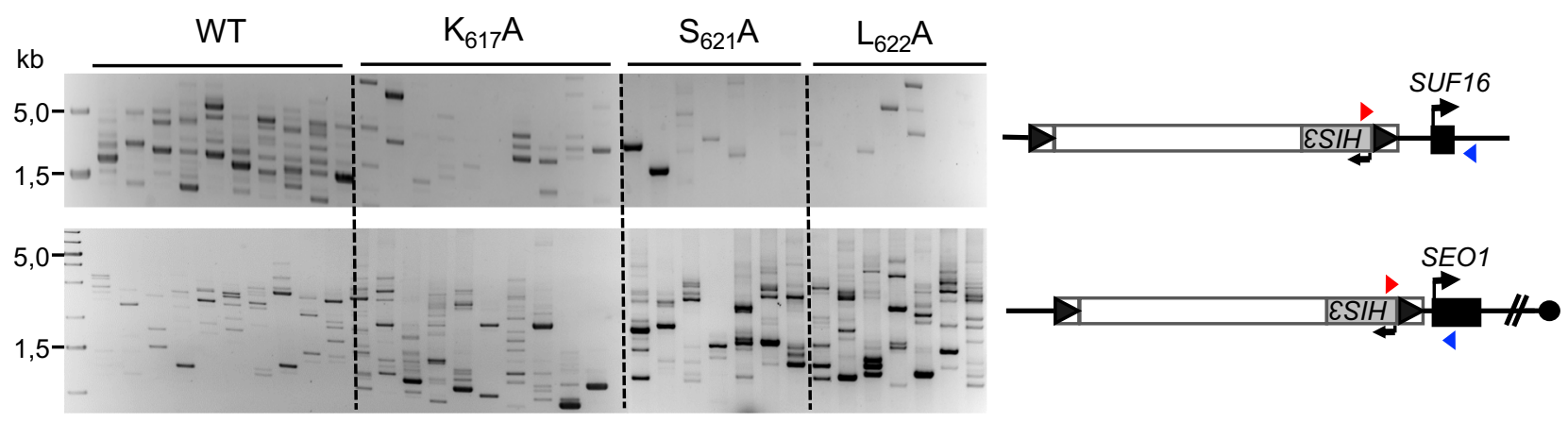

B

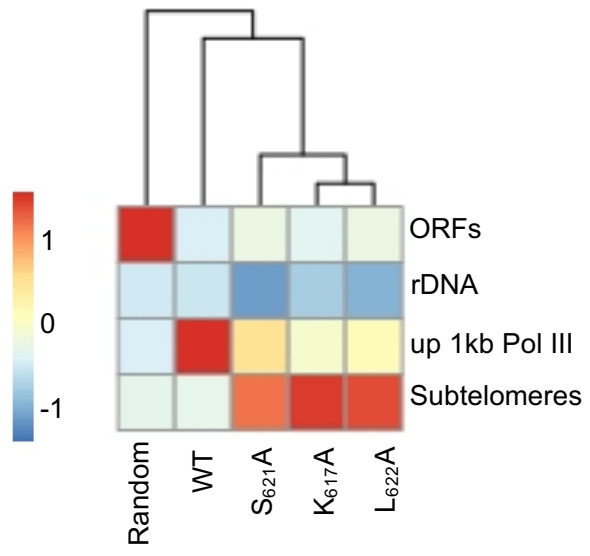

D

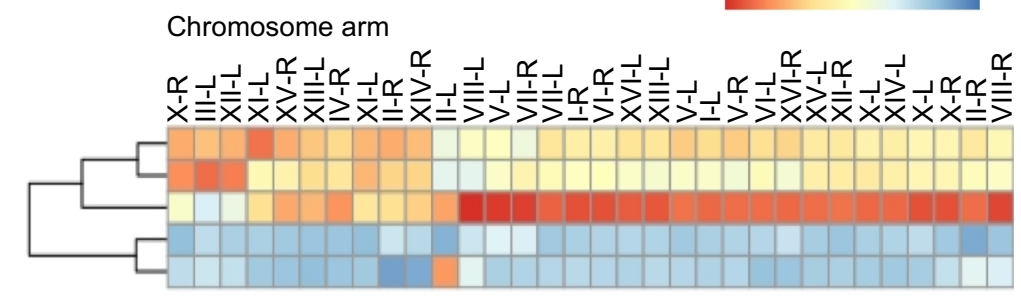

C

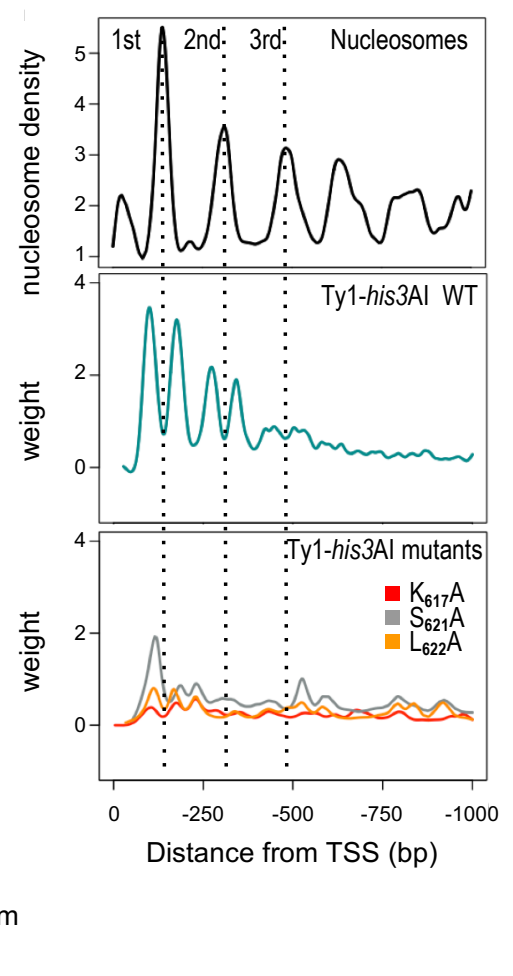

E

Chr V left subtelomere

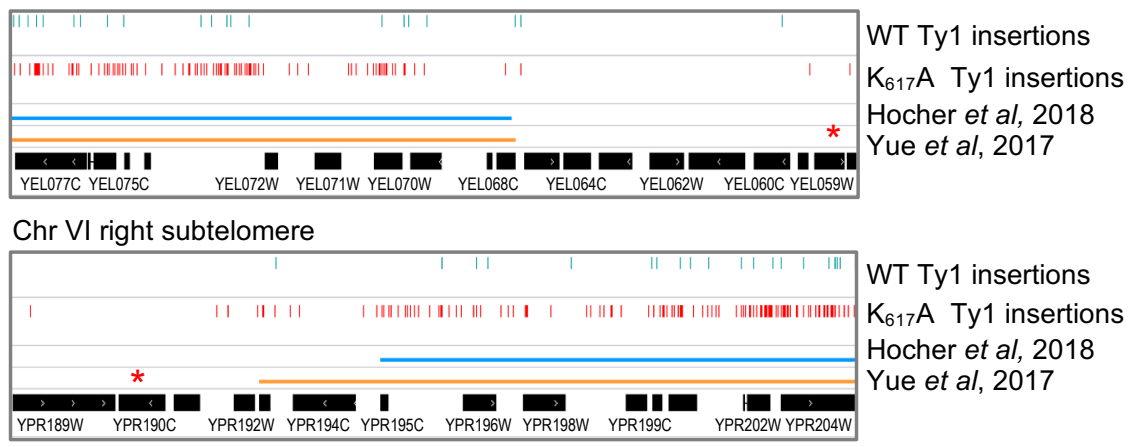


Figure 5. The IN1 bNLS targets Ty5 integration at Pol III-transcribed genes.

A

\begin{tabular}{|c|c|c|}
\hline IN1 (635 AA) & & \\
\hline \begin{tabular}{|l|l} 
NTD & CCD \\
\end{tabular} & CTD & \\
\hline IN5 (644 AA) & & 578 \\
\hline \begin{tabular}{|l|l|} 
NTD & CCD \\
\end{tabular} & CTD & \\
\hline
\end{tabular}

IN5 ${ }_{\triangle T D}(638 \mathrm{AA})$

\begin{tabular}{|l|l|l|}
\hline NTD & CCD & CTD \\
\hline
\end{tabular}

IN5 ${ }_{\Delta \mathrm{TD}+\mathrm{bNLS}}(673 \mathrm{AA})$
\begin{tabular}{|l|l|l|}
\hline NTD & CCD & CTD \\
\hline
\end{tabular}

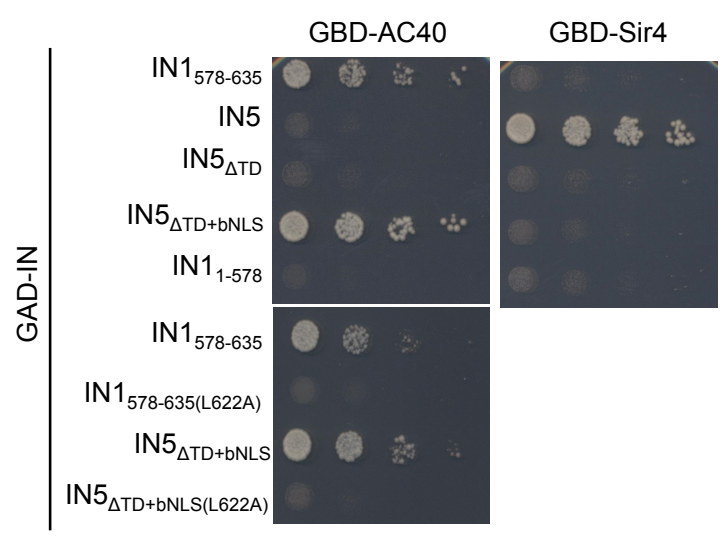

B
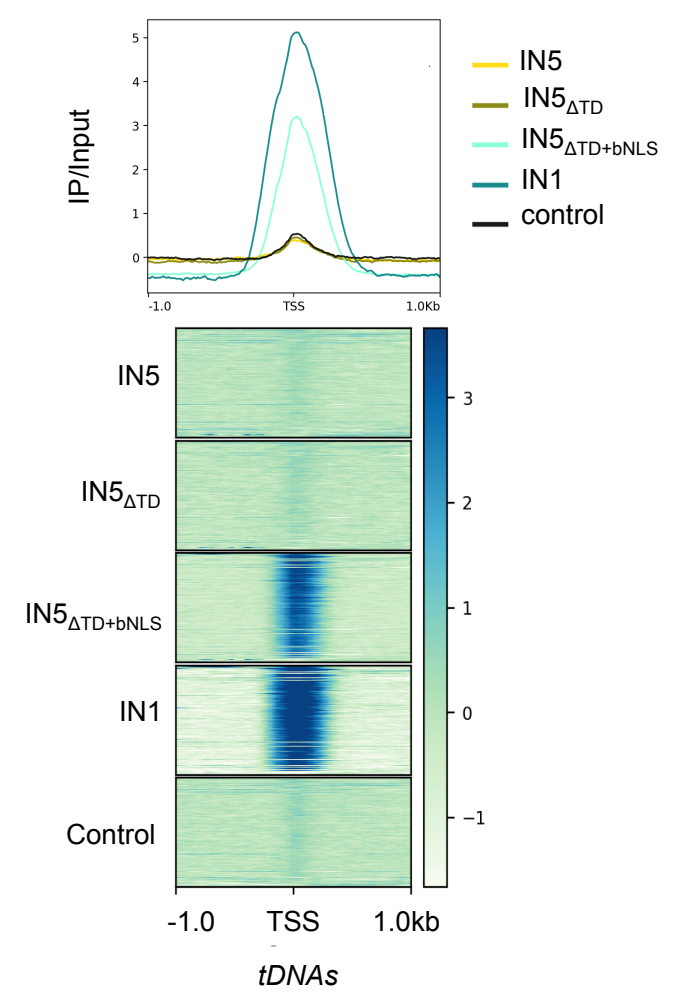

$\mathrm{D}$
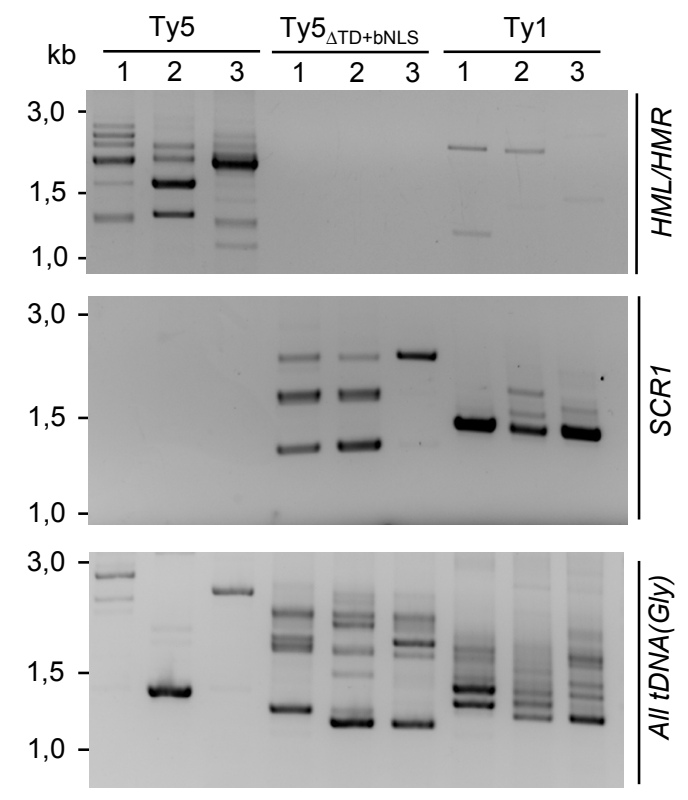

C

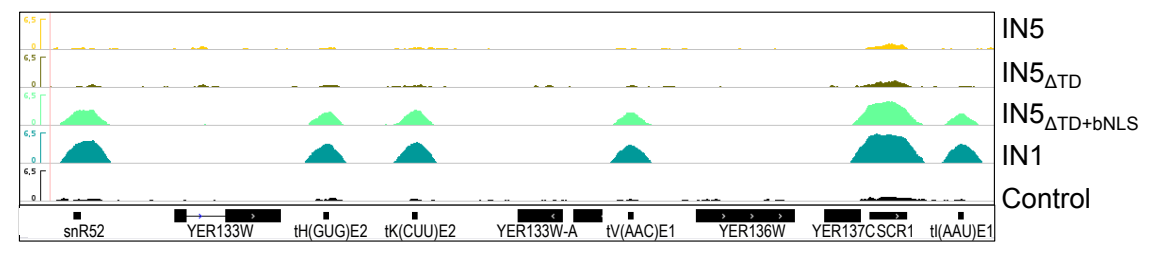


Figure 6. Model coupling the nuclear entry of Ty1 pre-integration conplex with Ty1 integration at Pol III-transcribed genes

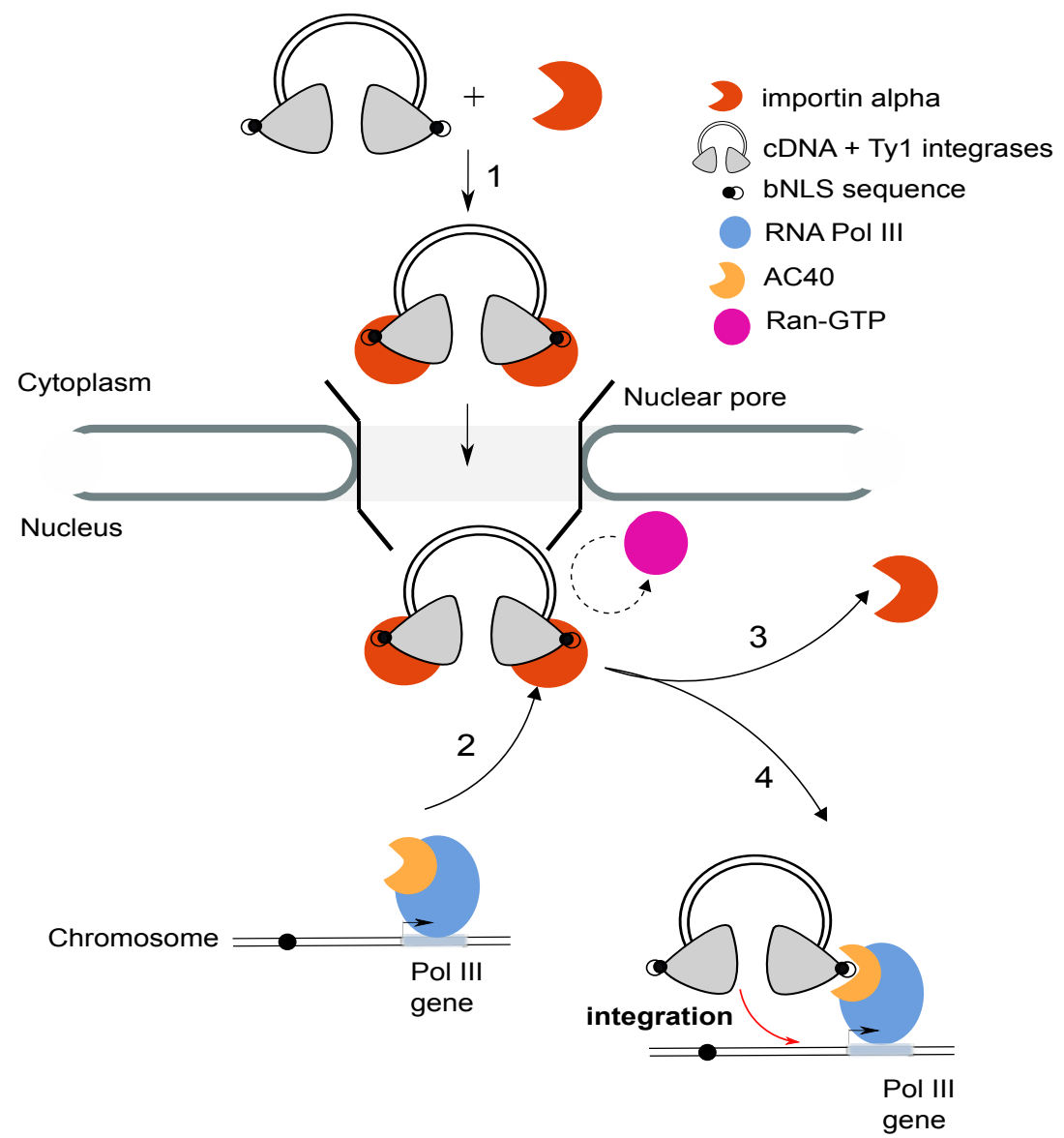

